\title{
Maternal inflammation has a profound effect on cortical interneuron development in a stage and subtype-specific manner
}

\author{
Navneet A. Vasistha $\mathbb{( D}^{1} \cdot$ Maria Pardo-Navarro ${ }^{1} \cdot$ Janina Gasthaus $\mathbb{D}^{1} \cdot$ Dilys Weijers $^{1} \cdot$ Michaela K. Müller $\mathbb{D}^{2}$. \\ Diego García-González ${ }^{1}{ }^{1}$. Susmita Malwade ${ }^{1}$ - Irina Korshunova ${ }^{1}$. Ulrich Pfisterer ${ }^{1}$. Jakob von Engelhardt ${ }^{2}$. \\ Karin S. Hougaard $\mathbb{D}^{3,4} \cdot$ Konstantin Khodosevich $^{1}$
}

Received: 15 August 2018 / Revised: 10 September 2019 / Accepted: 24 September 2019 / Published online: 8 October 2019

(c) The Author(s) 2019. This article is published with open access

\begin{abstract}
Severe infections during pregnancy are one of the major risk factors for cognitive impairment in the offspring. It has been suggested that maternal inflammation leads to dysfunction of cortical GABAergic interneurons that in turn underlies cognitive impairment of the affected offspring. However, the evidence comes largely from studies of adult or mature brains and how the impairment of inhibitory circuits arises upon maternal inflammation is unknown. Here we show that maternal inflammation affects multiple steps of cortical GABAergic interneuron development, i.e., proliferation of precursor cells, migration and positioning of neuroblasts, as well as neuronal maturation. Importantly, the development of distinct subtypes of cortical GABAergic interneurons was discretely impaired as a result of maternal inflammation. This translated into a reduction in cell numbers, redistribution across cortical regions and layers, and changes in morphology and cellular properties. Furthermore, selective vulnerability of GABAergic interneuron subtypes was associated with the stage of brain development. Thus, we propose that maternally derived insults have developmental stage-dependent effects, which contribute to the complex etiology of cognitive impairment in the affected offspring.
\end{abstract}

\section{Introduction}

Development of the fetal brain is highly influenced by the maternal environment. A multitude of factors, such as maternal nutrition, stress, hormonal imbalance, as well as the

These authors contributed equally: Navneet A. Vasistha, Maria PardoNavarro

Supplementary information The online version of this article (https:// doi.org/10.1038/s41380-019-0539-5) contains supplementary material, which is available to authorized users.

Konstantin Khodosevich

konstantin.khodosevich@bric.ku.dk

1 Biotech Research and Innovation Centre (BRIC), Faculty of Health, University of Copenhagen, Copenhagen N, Denmark

2 Institute of Pathophysiology, University Medical Center of the Johannes Gutenberg University Mainz, Mainz, Germany

3 National Research Centre for the Working Environment, Copenhagen $\varnothing$, Denmark

4 Institute of Public Health, University of Copenhagen, Copenhagen K, Denmark maternal immune status, play key roles in shaping normal brain development [1]. Maternal inflammation is known to increase the risk for severe psychiatric disorders including schizophrenia, bipolar disorder, intellectual disability, anxiety, autism spectrum disorders and cerebral palsy $[2,3]$ with high societal costs [4]. Although the exact mechanism of adverse neurodevelopment upon maternal inflammation is not known, data from animal experiments and clinical observations suggest that the cytokine-related pro-inflammatory response in the mother contributes to disordered development of the fetal brain and predisposes the offspring to additional stressors during postnatal maturation of the brain [5-7].

Cortical GABAergic interneurons arise during embryogenesis in the ganglionic eminences (GEs). Two of the three classes of GABAergic interneurons are generated in the medial ganglionic eminence (MGE), i.e., the parvalbumin (PV)- and somatostatin (SST)-expressing interneuron types, whereas the third class is generated in the caudal ganglionic eminence (CGE) and gives rise to a highly heterogeneous group of neurons that express serotonin receptor 3A (5HT3AR) and consists of vasoactive intestinal polypeptide (VIP), neuropeptide $\mathrm{Y}$, reelin-positive and other subtypes [8-11]. In mice, cortical GABAergic interneurons 
are produced during middle-late gestation (E9.5-E18.5) $[12,13]$, and migrate tangentially from the GEs to the cortical plate, where they start migrating radially into prospective cortical layers $[14,15]$. Subsequently, a significant proportion of interneurons undergoes programmed cell death $[16,17]$, while the surviving cortical interneurons mature over 2-3 months in mice [9].

Excitation-inhibition imbalance in the brain has been proposed as a major factor underlying the behavioral outcomes and cognitive decline associated with various neurodevelopmental disorders, including those associated with maternal inflammation [18-21]. Indeed, several studies show changes in activity and distribution of GABAergic interneurons in the cortex and hippocampus of the adult mouse brain upon maternal inflammation [22-24]. Such abnormalities are thought to occur due to perturbed brain development during early fetal or juvenile periods. Importantly, the development of neuronal circuits in the brain continues until late adolescence, up to 60-70 days postnatally in rodents and 20-25 years in humans [25]. Despite being acute, maternal inflammation can have long-lasting effects on several critical developmental processes such as precursor cell proliferation, neuronal migration and differentiation during embryogenesis, as well as postnatal neuronal maturation including neuronal survival, dendritic, synaptic, and axonal pruning and synaptogenesis. Some studies have addressed the developmental impairment of principal cortical neurons upon maternal inflammation [6, 26, 27], but surprisingly little is known regarding how development and maturation of cortical GABAergic interneurons are affected, and more importantly how those defects of GABAergic interneurons that are observed in adult animals [23, 24, 28] arise during brain development. Abnormal development of cortical inhibitory circuits can have a significant impact on animal behavior leading to phenotypes resembling human psychiatric disorders as has been shown for a number of genetic mouse models [29-31]. Furthermore, as cortical GABAergic interneurons represent a diverse class of neurons with more than 20 subtypes [32], various subtypes of GABAergic interneurons might be differentially affected by maternal inflammation contributing to the complex behavioral abnormalities of the offspring.

To study the impairment of cortical interneuron development due to maternal inflammation, we utilized a mouse model of maternal inflammation that involved injecting polyriboinosinic-polyribocytidylic acid (poly I:C), a synthetic polynucleotide that mimics viral double-stranded RNA, at embryonic day 9.5 (E9.5). This early time point is equivalent to the first-trimester period in humans and represents a period of high vulnerability to neurodevelopmental disorders caused by maternal infections [33]. It also marks the onset of cortical GABAergic interneuron production from the MGE in mice [34] as well as the pioneer Cajal-Retzius cells [35]. Poly I:C injection is a well-established model of maternal inflammation triggering an acute inflammatory response by activating the pro-inflammatory cytokines including IL-2, IL-6, IL-8, IL-1 $\beta$, IL-17a, and TNF $\alpha[6,36]$. It has been shown that these cytokines do not activate the embryonic microglia suggesting that maternal inflammation directly perturbs neuronal development $[37,38]$. Offspring born to poly I:C-injected mice show marked behavioral deficits, such as decreased prepulse inhibition (PPI), altered social interaction, and cognitive decline $[39,40]$. Thus, to understand the effect of maternal inflammation on interneuron development, we undertook a detailed analysis of embryos and pups exposed to maternal inflammation. We found poly I:C to have an acute effect on GABAergic interneuron precursor proliferation, migration, positioning, and maturation of GAD + neuroblasts. The effect of maternal inflammation was interneuron subtype-specific and demonstrated differential vulnerability of interneuron subtypes to mother-derived insults.

\section{Materials and methods}

\section{Animal breeding and genotyping}

All animal experiments were conducted in accordance with the guidelines of the National Animal Ethic Committee of Denmark. C57BL/6J (Janvier Labs), PV ${ }^{\text {Cre }}$ (017320, Jackson Labs), Ai9-tdTomato (007905, Jackson Labs) and GAD67-EGFP $\left(\operatorname{Gad} 1^{\text {tm1.1Tama }}\right)$ [41] mice were used in this study. The mice were kept in IVC cages and housed under a reversed light cycle with food and water ad libitum. Heterozygous GAD67-EGFP mice were bred with wild-type mice. Females were kept with males until the vaginal plug was detected, and then males were separated. Date of the vaginal plug were treated as E0.5, and embryos and pups were timed accordingly. Pups were weaned at P21 and littermates of the same sex were kept separately at 3-6 animals per cage.

EGFP + animals were identified by PCR using the Accustart II Polymerase (QuantaBio). PCR conditions used were: $94{ }^{\circ} \mathrm{C}$ for $2 \mathrm{~min}$, followed by 35 cycles of $94^{\circ} \mathrm{C}$ for $15 \mathrm{~s}, 62^{\circ} \mathrm{C}$ for $30 \mathrm{~s}, 72^{\circ} \mathrm{C}$ for $30 \mathrm{~s}$, and final extension at $72^{\circ} \mathrm{C}$ for $5 \mathrm{~min}$. The following primers (in $5^{\prime}-3^{\prime}$ orientation) amplified a $345 \mathrm{bp}$ region.

\section{EGFP_F CCTACGGCGTGCAGTGCTTCAGC EGFP_R CGGCGAGCTGCACGCTGCGTCCTC}

\section{Induction of maternal immune activation using poly I:C}

Commercially prepared poly I:C (Sigma, P9582) was purchased and dissolved in phosphate buffered-saline (PBS) to give a $1 \mathrm{mg} / \mathrm{ml}$ stock solution. Two lots of poly I:C were 
used during the course of this study and no lot-specific effects of poly I:C were observed (Supplementary Fig. 1 and Supplementary Table 1).

Pregnant females at E9.5, E12.5, and E16.5 received a single-tail intravenous injection of poly I:C equivalent to 5 $\mathrm{mg} / \mathrm{kg}$ body weight under mild restraint. Control females received an equal volume of PBS (no randomization regarding assignment to PBS or poly I:C injection). Information regarding rates of abortion and size of litters for poly I:C and PBS-injected mice, as well as number of litters, animals and females/males used for each experiment is shown in Supplementary Tables 2 and 3. Lack of litterspecific effects for PBS- or poly I:C injections are shown in Supplementary Fig 2 and Supplementary Table 4.

For experiments involving 5-bromo-2'-deoxyuridine (BrdU) labeling of progenitors, $50 \mathrm{mg} / \mathrm{kg}$ of BrdU (Sigma, B5002) was chosen as in our previous studies [42, 43] and was injected intraperitoneally (i.p.) into dams at E10.5, E14.5, and E17.5. Dams were sacrificed $2 \mathrm{~h}$ after labelling and embryo were dissected and their heads collected in $4 \%$ paraformaldehyde (PFA) solution.

\section{Plasma IL-6 estimation}

Three hours post poly I:C or PBS injection, blood samples were taken from the tail vein in EDTA precoated Eppendorf tubes. Following centrifugation ( $3000 \mathrm{rpm}, 10 \mathrm{~min})$, the supernatant was collected in a separate tube and stored at $-80^{\circ} \mathrm{C}$ when all samples were analyzed together. A mouse IL-6 DuoSet ELISA kit (R\&D Systems) was used to measure IL-6 levels. Absorbance was measured at 450 and 560 $\mathrm{nm}$ using a Glomax microplate reader (Promega). For analysis, readings at $560 \mathrm{~nm}$ were subtracted from those at $450 \mathrm{~nm}$ and normalized to blank controls. Values were obtained by linear regression of a plot of concentrations of known standards versus normalized absorbance values.

\section{Mouse behavior}

PBS- and poly I:C-treated offspring (P35, male and female) were first acclimatized to the room and subsequently placed in the center of a circular open field (OF) of $1 \mathrm{~m}$ diameter. Movement was recorded for $10 \mathrm{~min}$ using the Noldus Ethovision XT video tracking system v5 (Noldus Information Technology). Time spent in the central zone $(0.7 \mathrm{~m}$ inner diameter) and periphery was extracted as were basic locomotor parameters such as total distance traveled, in total and in 1-min intervals and mean velocity.

Social interaction was tested immediately after the openfield test. For this, two rectangular wire containers (6.2 $\mathrm{cm} \times 7.8 \mathrm{~cm} \times 12.4 \mathrm{~cm}, \mathrm{H} \times \mathrm{W} \times \mathrm{L}$ ) large enough to hold a mouse and allowing for social interaction were placed equidistant from the center of the OF. An unknown mouse was alternated between the two containers with the other kept empty. A region of $10 \mathrm{~cm}$ around the containers was treated as the social interaction zone, the remaining part of the arena as a nonsocial zone. Time spent by control and poly I:C mice in the social and nonsocial zones was extracted via Ethovision.

Acoustic startle reaction and PPI were tested at 5 weeks of age as described [44] in two chambers (San Diego Instruments, San Diego, USA) with $70 \mathrm{~dB}(\mathrm{~A})$ white background noise. A piezoelectric accelerometer transduced displacement of mouse test tubes $(\varnothing 3.6 \mathrm{~cm})$ in response to movements of the animal. Animals were acclimatized for $5 \mathrm{~min}$ in the tube before sessions started and ended with five startle trials of $40 \mathrm{~ms} 120 \mathrm{~dB}(\mathrm{~A})$ bursts of white noise. In between, 35 trials were delivered in semirandomized order (ten trials of $120 \mathrm{~dB}(\mathrm{~A})$; five each of four prepulse + startle trials (prepulses of $72,74,78$, and $86 \mathrm{~dB}(\mathrm{~A})$ ); five trials with only background noise). Tube movements were averaged over $100 \mathrm{~ms}$ following onset of the startle stimulus (AVG). The five AVGs for each prepulse intensity were averaged and used to calculate PPI, which was expressed as percent reduction in averaged the prepulse AVGs compared with the average of the ten middle startle trials: \%PPI = $[1-($ Prepulse + pulse/Pulse $)] \times 100$.

\section{Perfusion, sectioning and immunohistochemistry}

All steps were performed at ambient temperature unless otherwise noted. A description of antibodies used in this study is given in Supplementary Table 5.

Postnatal mice were anaesthetized with a combination of xylazine and ketamine injected i.p. This was followed by transcardial perfusion initially with cold PBS to flush out blood and then with cold 4\% PFA. Brains were then removed and postfixed in $4 \%$ PFA overnight at $4{ }^{\circ} \mathrm{C}$ before being stored in PBS with $0.01 \%$ sodium azide.

Brains were sectioned using a vibrating microtome (Leica, VT1000S) at a thickness of $50 \mu \mathrm{m}$ and stored in PBS with $0.01 \%$ sodium azide at $4{ }^{\circ} \mathrm{C}$. E10.5-E17.5 embryos were fixed in 4\% PFA overnight, dehydrated in $30 \%$ sucrose and sectioned at $25 \mu \mathrm{m}$ thickness using a cryostat (Leica CM3050).

For staining, sections were blocked and permeabilized with $3 \%$ BSA (in $0.2 \%$ Triton X-100 containing PBS). Subsequently, they were incubated overnight at $4{ }^{\circ} \mathrm{C}$ with appropriate primary antibodies. The following day, sections were washed and incubated with Alexa Fluor conjugated secondary antibodies for $2 \mathrm{~h}$ at room temperature. Nuclei were counterstained with DAPI (Sigma) and coverslips were mounted on slides with FluorSave (Merck).

BrdU labeling was done similar as before [45] by treating with $1 \mathrm{M} \mathrm{HCl}$ at $37^{\circ} \mathrm{C}$ for $30 \mathrm{~min}$, followed by neutralization with $10 \mathrm{mM}$ Tris- $\mathrm{HCl}$ (pH8.5), and antibody labelling 
was carried out as described above. For BrdU and Nkx2.1/ COUP-TFII double-labelling, antigen retrieval was performed before BrdU labeling using sodium citrate solution $(10 \mathrm{mM})$ at $85^{\circ} \mathrm{C}$ for $15 \mathrm{~min}$.

\section{Image acquisition and analysis}

Images were acquired using a confocal microscope (Leica SP8, Leica Microsystems) and analyzed using ImageJ (NIH) and Imaris (Bitplane AG). After correcting for brightness and contrast, figures were prepared using Adobe Illustrator (Adobe Inc). Graph preparation and statistical analysis were carried out using Prism 7.0 (GraphPad).

Distribution of GABAergic interneurons across the cortex was analyzed in the region of interest (ROI) spanning from the upper edge of the corpus callosum or the subventricular zone (SVZ) to the pia at P1-60 old mice or E14.5-E17.5-old mice, respectively, and having 200-400 $\mu \mathrm{m}$ in width (medial-lateral) depending on age. The whole length of ROI was subdivided into ten equal bins for E14.5P9 ages, and in six layers at P15-60 when cortical layering could be distinguished based on DAPI staining. Presumptive motor and somatosensory regions in the developing brain are referred to as motor and somatosensory cortex, respectively.

The direction of migration at E17.5 was determined in the intermediate zone (IZ) and cortical plate using ImageJ according to the direction of the major neurite expanding from the cell body of the neuroblast, and the angle of migration was measured between the vertical axis (from the ventricle to the pia) and the axis of the major neurite.

\section{Electrophysiology}

Acute brain slices were prepared from P53-57 old mice. To determine an appropriate n-number, we calculated before performing the experiments the required sample size to reach significance $(p<0.05)$ if the observed effect was more than $20 \%$. The power analysis was based on means and distributions of data from our previous electrophysiological experiments. Using $G^{*}$ Power for this analysis $(\alpha$-error $=$ 0.05 ; power $(1-\beta$ error $)=0.85)$, we determined that $n$ number of 17 per group as required. We recorded from a slightly higher number of cells (26 cells from three PBSinjected mice and 25 cells from four poly I:C-injected mice), and 2-3 neurons were recorded per brain slice. Only basket cells were recorded and few chandelier cells were excluded from the analysis. To this end, mice were deeply anesthetized with Isoflurane (3\%) and the brains quickly removed and dissected in ice-cold sucrose-containing ACSF ( $212 \mathrm{mM}$ sucrose, $0.02 \mathrm{mM} \mathrm{CaCl}, 7 \mathrm{mM} \mathrm{MgCl}, 3 \mathrm{mM}$ $\mathrm{KCl}, \quad 1.25 \mathrm{mM} \quad \mathrm{NaH}_{2} \mathrm{PO}_{4}, \quad 26 \mathrm{mM} \quad \mathrm{NaHCO}_{3}, \quad 10 \mathrm{mM}$
D-glucose oxygenated with carbogen). Two hundred and fifty micrometers thick coronal brain slices were cut with the help of a tissue slicer (Leica VT1200S, Wetzlar, Germany) in ACSF (25 mM D-glucose, $125 \mathrm{mM} \mathrm{NaCL}$, $1.25 \mathrm{mM} \mathrm{NaH}_{2} \mathrm{PO}_{4}, 26 \mathrm{mM} \mathrm{NaHCO}_{3}, 2.5 \mathrm{mM} \mathrm{KCl}, 2 \mathrm{mM}$ $\mathrm{CaCl}_{2}, 1 \mathrm{mM} \mathrm{MgCl}{ }_{2}$ oxygenated with carbogen).

Brain slices were visualized under an upright Olympus BX51WI microscope fitted with a $\times 40$ water-immersion objective (LUMPlan FI/IR, NA 0.8w; Olympus, Japan) with infrared optics. Whole-cell recordings were obtained from single neurons in layer $2 / 3$ of the somatosensory cortex under visual guidance. On average, 2-3 neurons were recorded per brain slice. Borosilicate glass pipettes (3-5 M $\Omega$ ) were pulled with a micropipette puller (Sutter Instruments, Novato, CA, USA) and filled with intracellular solution (130 mM K-gluconate, $10 \mathrm{mM}$ HEPES, $10 \mathrm{mM}$ phosphocreatinine- $\mathrm{Na}, 10 \mathrm{mM}$ Na-gluconate, $4 \mathrm{mM}$ ATP$\mathrm{Mg}, 0.3 \mathrm{mM}$ GTP, $4 \mathrm{mM} \mathrm{NaCl}$; pH 7.2). Intrinsic membrane properties and firing patterns were recorded in current clamp mode with $50 \mathrm{pA}$ current steps ranging from -50 to $900 \mathrm{pA}$. Intrinsic membrane properties and firing properties were analyzed with IGOR Pro (WaveMetrix, USA) and Microsoft Excel (Microsoft, USA).

\section{Western blot}

Mice were euthanized by cervical dislocation and brains were quickly removed. The cortex was dissected in PBS on ice and stored at $-80^{\circ} \mathrm{C}$ until use. Cortices were homogenized using a Dounce tissue grinder set (Loose followed by Tight pestle, Sigma) in ice-cold $300 \mu \mathrm{l}$ RIPA buffer supplemented with Complete EDTA-free Protease inhibitor (Roche). Lysates were incubated for $10 \mathrm{~min}$ at $4{ }^{\circ} \mathrm{C}$ and collected by centrifugation at $5000 \times g$ for 10 min. Protein concentration was determined using the bicinchoninic acid kit (Pierce) and BSA as a standard. Equal amount of SDS sample buffer, Laemmli $2 \times$ concentrate (Sigma) was added to each lysate and boiled for $5 \mathrm{~min}$. Samples were separated by $15 \%$ SDS-PAGE gel and transferred onto the $0.45 \mu \mathrm{m}$ pore size Immobilone-FL Membrane (Millipore) for $1 \mathrm{~h}$ at $20 \mathrm{~V}$. Membranes were blocked in 3\% BSA (Applichem) in TBS-T (TBS plus $0.1 \%$ Tween 20) for $1 \mathrm{~h}$ at room temperature, and then incubated overnight at $4{ }^{\circ} \mathrm{C}$ with primary antibody: mouse anti-GAPDH (1:5000, Abcam) or rabbit anti-PV (1:10000, Swant) diluted in blocking solution. After washing with TBS-T, membranes were incubated with secondary horse anti-mouse HRP-conjugated (1:20,000; Vector laboratories) or anti-rabbit HRP-conjugated (1:20,000; GE Healthcare) in blocking solution for $1 \mathrm{~h}$ at room temperature. Detection was performed using the ECL chemiluminescence reagent (Pierce) and X-ray films (AGFA). 


\section{Statistical analysis}

Statistical analysis of the data was performed with Prism (GraphPad software, USA). Normality of data distribution was analyzed by D'Agostino and Shapiro-Wilk's tests. Normally distributed data were analyzed using the Student's $t$-test (for two groups) or by one-way ANOVA test (for more than two groups). Welch's correction was applied when standard deviations of the two groups differed from each other. For simultaneous comparison of two parameters between two and more groups, we used two-way ANOVA and the Bonferroni post-hoc test. Equality of variances was analyzed using Dunnett's test. A linear mixed model (LMM) analysis was used to analyze electrophysiology results. We extended the LMM analysis to address the issue of a shared intrauterine environment in multiparous species [46], especially applying it to outcomes of the histological analysis (Supplementary Table 6). For multiple comparisons, $p$ values from LMM analysis were corrected with Benjamini-Hochberg method.

To determine an appropriate number of animals, we estimated sample size based on our previous experiments and on similar experiments published by other groups. The investigators were not blinded to allocation during experiments and outcome assessment. Females and males were analyzed together, and all the graphs represent joint sex data unless otherwise stated. For all behavior experiments, sexrelated differences were analyzed by two-way ANOVA with exposure and sex as factors.

Basal startle and habituation to the first five $120 \mathrm{~dB}$ noise bursts and PPI were analyzed by two-way ANOVA with poly I:C exposure and sex as factors; for basal startle habituation with the first five 120 trials as the repeated measure, for PPI with prepulse level $(72,74,78$, and $86 \mathrm{~dB}$ (A)) as the repeated measure.

\section{Results}

\section{Maternal inflammation model in transgenic mice that labels all cortical GABAergic interneurons}

In order to reveal how the development of cortical GABAergic interneurons is affected by maternal inflammation, we utilized the GAD67-EGFP knock-in transgenic mouse line that labels all GABAergic interneurons in the cortex by EGFP [41]. Maternal inflammation was induced by injecting poly I:C in pregnant dams at E9.5 corresponding to the early first trimester of pregnancy in humans; [47] a period of highest risk of induction of neurodevelopmental disorders caused by maternal infections [33]. This period also marks the onset of cortical GABAergic interneuron production in mice, which starts in the MGE at

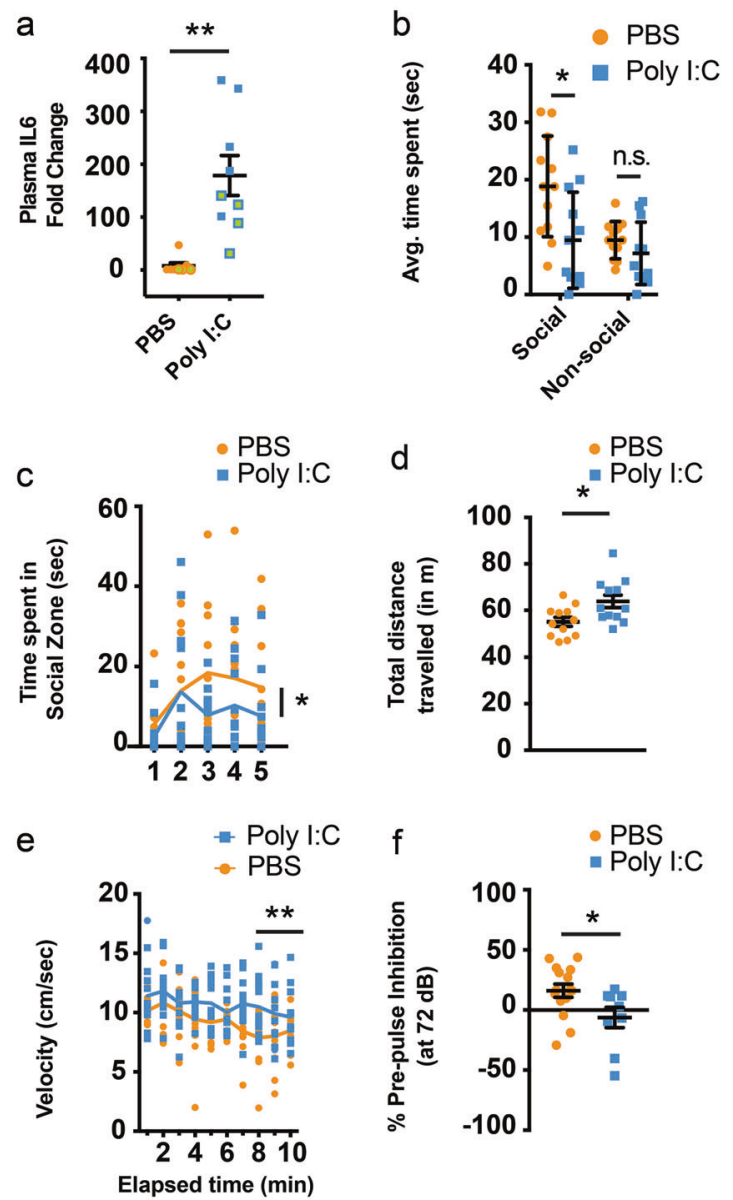

Fig. 1 Poly I:C induces rapid upregulation of IL-6 in maternal plasma and behavioral impairments in maternal inflammation-affected offspring. a Upregulation of IL-6 protein in maternal plasma $3 \mathrm{~h}$ after i.v. injection of poly I:C ( $n=8$ and 9 for PBS and poly I:C respectively, 5 wild-type and 3 and 4 GAD67-EGFP mice, respectively, labeled by filled and green circles/squares, respectively). Prenatal exposure to maternal inflammation results in impaired social zone preference (b) $(n=12$ each), as well as a decrease in time spent in social zone (c) $(F=3.23)$. Mice exposed to maternal inflammation during gestation present signs of hyperactivity as seen by total distance traveled (d) $(n=12$ each) and speed of running over a $10 \mathrm{~min}$ period (e) $(n=12$ each). f Maternal inflammation during early gestation induces schizotypal effects in offspring that can be gauged by a decreased prepulse inhibition (at $72 \mathrm{~dB})$ ( $n=15$ and 9 for PBS and poly I:C, respectively). Comparison of means by Student's $t$-test in $\mathbf{a}, \mathbf{d}$, and $\mathbf{f}, t$-test with Holm-Sidak correction for multiple comparison in $\mathbf{b}$ and two-way ANOVA in $\mathbf{c}$ and, $\mathbf{e}$ (mean \pm SEM are shown, $* p<0.05, * * p<0.005)$

E9.5 [34]. We confirmed the induction of inflammation by an increase in maternal plasma IL-6 levels $3 \mathrm{~h}$ after injection of poly I:C both in wild-type and EGFP + females (Fig. 1a). To validate that poly I:C mouse model in our hands has a similar functional impact on the offspring as previously described [40,48], we performed behavioral experiments on control and poly I:C-affected offspring testing for social interaction and memory, OF and startle (including PPI) behavior. Control mice on average spent significantly more 


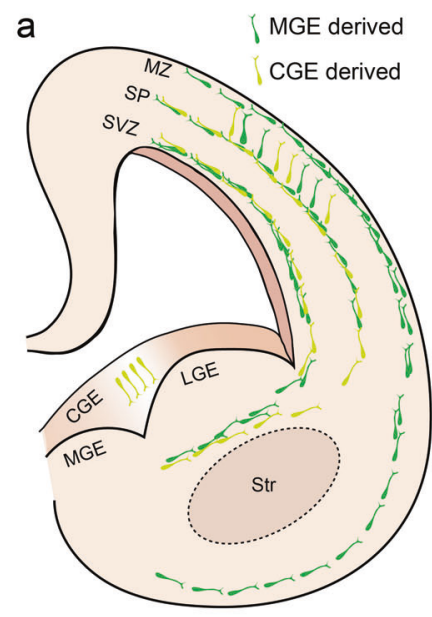

d
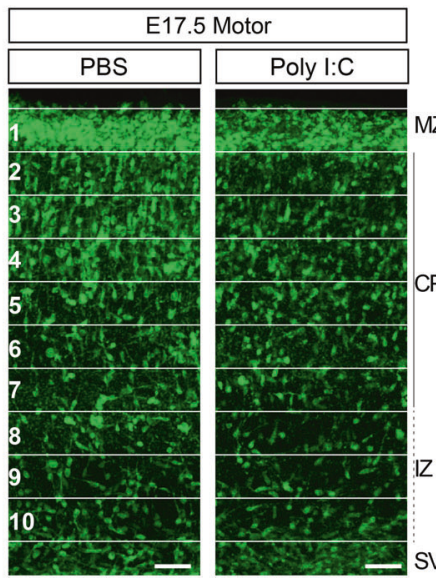
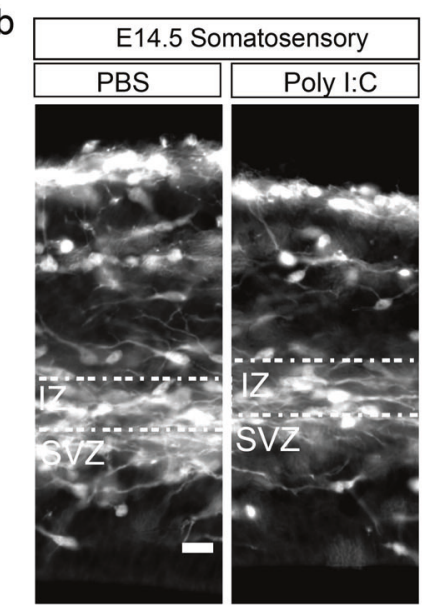

d'

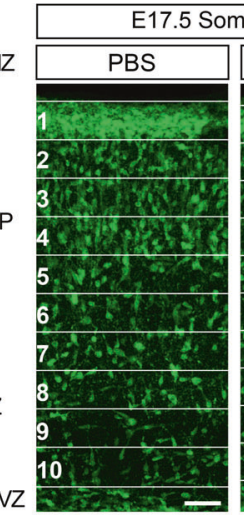

Fig. 2 Early migratory deficits of GAD-EGFP + neuroblasts due to maternal inflammation. a A schematic overview of the migratory routes taken by $\mathrm{GAD}+$ neuroblasts during embryonic development $[57,58]$. b, c Reduction in the density of EGFP+ neuroblasts migrating into the cortical intermediate zone at E14.5 as a result of maternal inflammation. Panel $\mathbf{b}$ shows somatosensory region of the developing cortex. Dotted box denotes the region of counting. Quantifications from the dotted boxes are shown in c (motor cortex: $n=8$ each; somatosensory cortex: $n=8$ each). d, e Superficial regions of the cortical column show a reduction in density of EGFP+ neuroblasts due to maternal inflammation. Cortical columns from developing motor (d) and somatosensory (d') cortical regions were divided into ten equal-sized bins and the number of EGFP+ cells counted in

time interacting with the unfamiliar mouse in comparison with the inanimate object (the empty container), while offspring affected by maternal inflammation did not have such a preference (Fig. 1b). Control offspring also interacted with the unfamiliar mouse for longer durations than those affected by the maternal inflammation (Fig. 1c). In addition, offspring affected by the maternal inflammation displayed signs of hyperactivity observed as an increase in the total distance traveled and in running speed in the open-

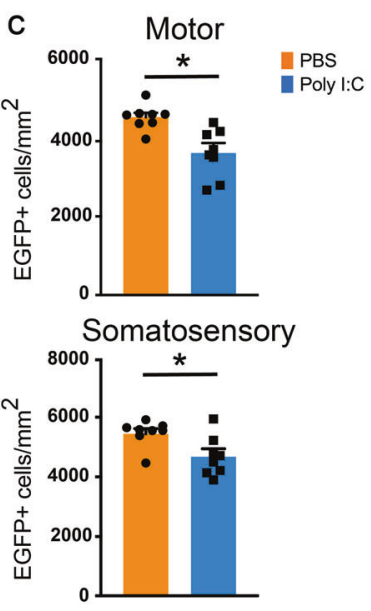

e

Motor
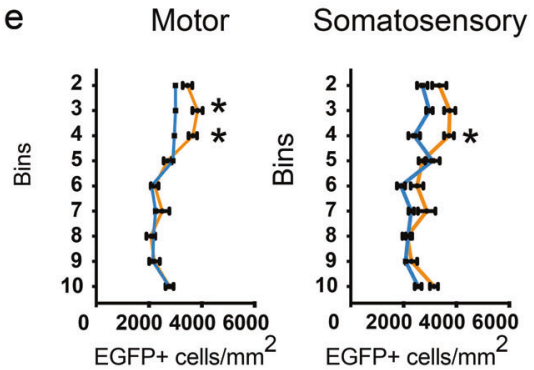$$
f
$$

each (with the exception of bin 1). Bin 10 represents the upper margin of the IZ. Densities in the graphs (e) show a reduction in bins 3-4. f Embryos exposed to maternal inflammation showed a decreased total density of migratory neuroblasts at E17.5. EGFP + cells were counted in the cortical plate and intermediate zone across bins $2-10 \quad(n=10$ each). $\mathbf{g}$ Radar plot showing that a greater percentage of neuroblasts in the cortical plate migrate laterally due to maternal inflammation as assessed by the angle of leading process. Angles were grouped into $30^{\circ}$ bins and relative percentages were plotted. Each spoke represents $10 \%$. Comparison of means by Student's $t$-test in $\mathbf{c}$, and $\mathbf{f}, t$-test with Holm-Sidak correction for multiple comparison in $\mathbf{e}$ (mean \pm SEM are shown, $* p<0.05, * * p<0.005)$. Scale bars: $50 \mu \mathrm{m}$

field test (Fig. 1d, e). For the basal startle reaction and the habituation to the first five $120 \mathrm{~dB}$ noise bursts, there were no statistically significant differences owing to poly I:C exposure; the startle reaction in males was significantly stronger than in females (data not shown). ANOVA analysis of PPI, with poly I:C exposure and sex as factors and level of prepulse intensity as the repeated measure $(72,74,78$, and $86 \mathrm{~dB}(\mathrm{~A})$ ), showed significant interaction between poly I:C exposure and prepulse level $(p=0.049)$, whereas 
interaction between prepulse level and sex had $p>0.05$ (there was also no three-way interaction between prepulse level, poly I:C exposure, and sex). Separate analysis of PPI at each prepulse level indicated that the statistically significant interaction was due to differences at the $72 \mathrm{~dB}$ prepulse level (Supplementary Fig. 3a), where exposed offspring showed reduced inhibition compared with control offspring (Fig. 1f). Overall, we did not observe marked differences in behavior between males and females (except for total distance traveled in the open-field test) (Supplementary Fig. 3b-j). Thus, maternal inflammation triggered by poly I:C injection led to impaired social behavior, increased anxiety and sensorimotor gating deficits in wild-type mice. These phenotypes were similar to those observed in previous studies of poly I:C-injected mice $[5,6,22,49-53]$.

\section{Maternal inflammation impacts early stages of cortical GABAergic interneuron development}

While the effect of maternal inflammation on offspring behavior has been well documented, there is little information about how maternal inflammation affects the development of inhibitory circuits in the cortex that might underlie abnormal animal behavior. In the subsequent analyses, we focused mainly on the developing somatosensory and motor cortices, since these are the best characterized cortical areas in mice that are evolutionarily conserved in humans and individuals with schizophrenia frequently show anatomical and functional impairments in motor and sensory areas [54-56]. We therefore investigated the effect of poly I:C (injected at E9.5) on the early stages of interneuron development. Cortical GABAergic interneurons originate between E9.5 and E18.5 in the GEs [12, 13] from where they migrate tangentially into the developing cortex. They enter the cortical plate at two principal sites-a superficial stream along the marginal zone just below the pia, and a deeper stream at the IZ/SVZ [57, 58]. Once in the developing cortical plate, GABAergic interneurons switch to a radial mode of migration and invade the cortical plate (Fig. 2a). Strikingly, we observed a clear effect of maternal inflammation on cortical GABAergic interneurons early during their development, since already at E14.5 fewer EGFP + neuroblasts were observed in the IZ of poly I:Cexposed embryos at the regions of the developing cortex corresponding to the motor and somatosensory cortices (Fig. 2b, c). In maternal inflammation-affected embryos, there were no evidence in disturbance of stream of neuroblasts migrating from the GE to the cortical plate, and we did not find large assembles of neuroblasts that were stuck during migration (Supplementary Fig. 4a). In addition, the density of EGFP + neuroblasts in the SVZ did not change in response to maternal inflammation (Supplementary Fig. 4b).
To study differences in migratory patterns of GABAergic interneurons, we chose E17.5 time point when the majority of interneurons reach the developing cortex and start to migrate radially within the cortex. We divided the cortex into ten equally sized bins (see "Materials and Methods" section) and quantified the number of EGFP+ neuroblasts in each. The thickness of the cortical plate, IZ, and MZ did not show any changes in E14.5 or E17.5 embryos due to exposure to maternal inflammation (Supplementary Fig. 4c, d). Remarkably, maternal inflammation resulted in largescale redistribution of GABAergic interneurons with an evident reduction in density of EGFP + neuroblasts in the regions corresponding to the motor and somatosensory cortices of the developing cortex at E17.5 (Fig. 2d, d'). Quantification of densities per bin showed that pups exposed to maternal inflammation had fewer EGFP+ neuroblasts in the superficial layers of the cortical plate (Fig. 2e, bins 3-4 for M1 and bin 4 for S1), which also resulted in a decrease in total neuroblast density across the cortical plate and IZ (Fig. 2f). However, while the developing somatosensory cortex showed an additional increase in percentage of EGFP+ neuroblasts stacked in the middle layers, a similar effect was not seen in the motor cortex (Fig. $2 d, d^{\prime}$, $\mathrm{e}$, bin 5), indicating a differential effect of maternal inflammation on different cortical areas.

To account for the effect on distribution of GAD+ neuroblasts caused by acute maternal inflammation we analyzed the directionality of neuroblast migration in the IZ and cortical plate at E17.5. While GAD+ neuroblasts in the cortex of control offspring predominantly migrated towards the pia, their direction in inflammation-affected cortices was significantly different with a greater fraction migrating laterally (Fig. $2 g$ and Supplementary Fig. 4e).

\section{Maternal inflammation impairs positioning of GABAergic interneurons in the developing cortex}

As interneuron migration continues until the second postnatal week, we chose to analyze whether these initially observed differences continue to affect the positioning of interneurons in the postnatal cortex. We hence studied several postnatal stages from P3 to P30 to understand how maternal inflammation can lead to a disordered arrangement of GABAergic interneurons in the mature cortex.

At P3 and P6, the difference in GAD+ cell density had amplified to cover the entire length of the cortical plate with the exception of the lowermost bins (bins 9-10, Fig. 3a, b, Supplementary Fig. 5a, b). Furthermore, the magnitude of the difference was consistently greater in the prospective somatosensory cortex than the motor cortex. Interestingly, the relative distribution of GAD + neuroblasts across bins was similar in pups exposed to PBS or poly I:C (Supplementary Fig. 5c, d). This suggests that the decrease in GAD+ 

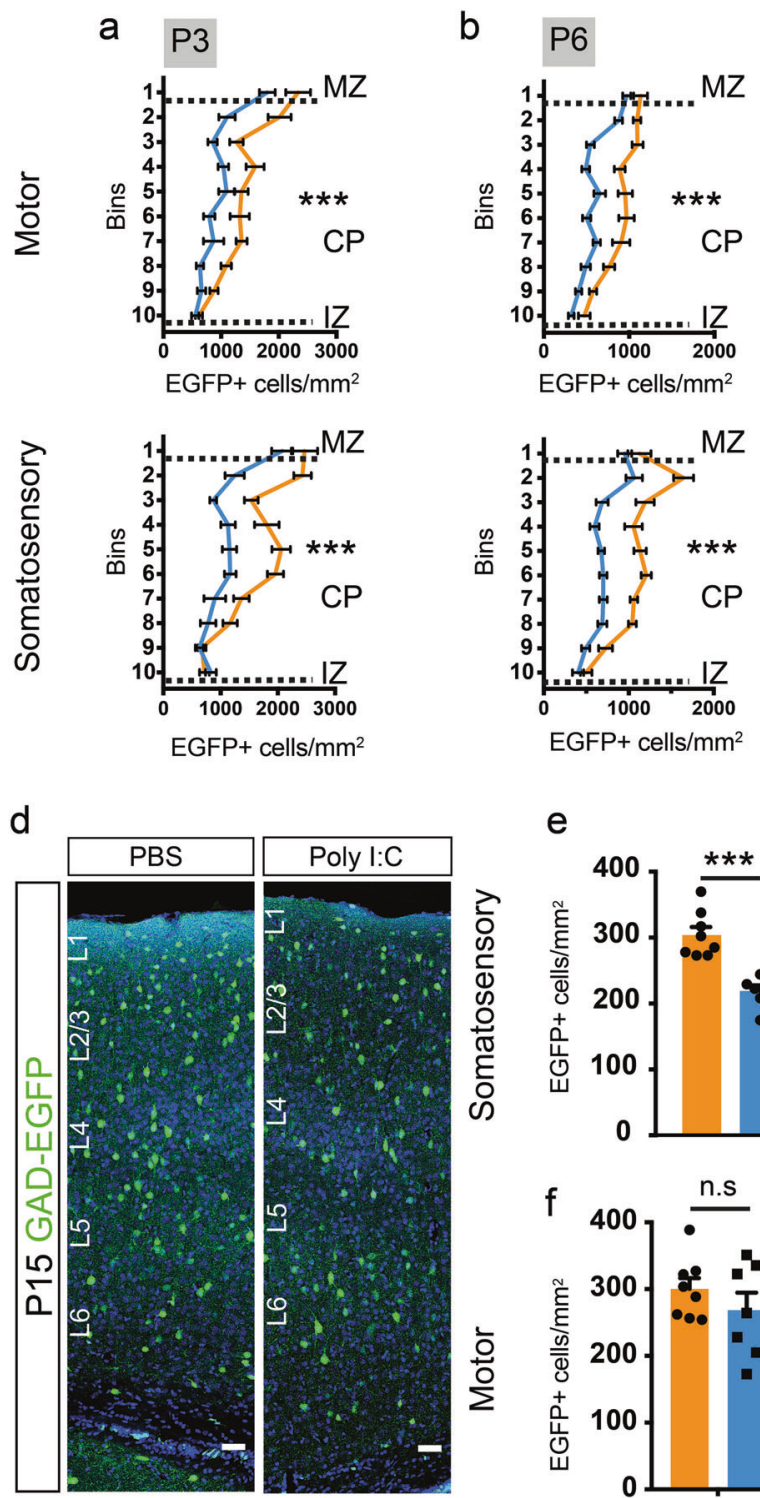

e
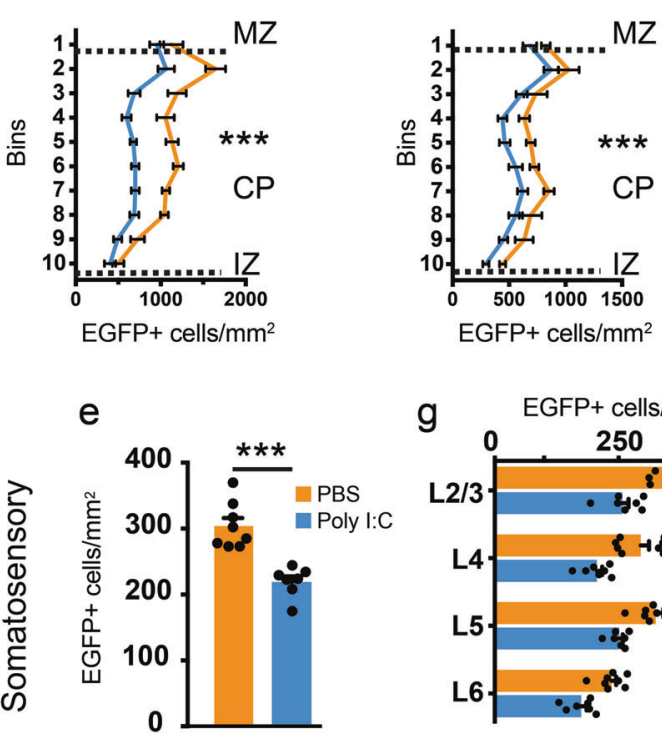

$9 \quad \mathrm{EGFP}+$ cells $/ \mathrm{mm}^{2}$
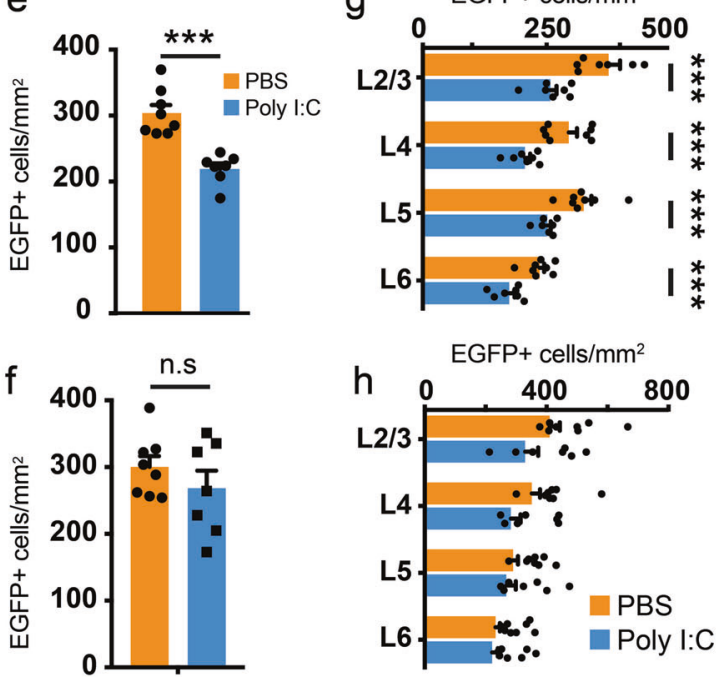

\section{P9}

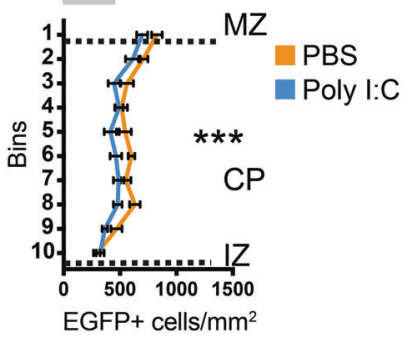

17
Fig. 3 Maternal inflammation causes large-scale impairment in the distribution of GAD-EGFP+ neuroblasts and mature interneurons in the cortex. a-c Graphs showing a decrease in density of EGFP+ cells measured by counting EGFP + cells in ten equally sized bins in the cortical plate of the presumptive motor and somatosensory cortices. Dotted lines represent the margins of IZ, CP, and MZ, respectively ( $\mathrm{P} 3$ motor: $F=21.85, \mathrm{P} 3$ somatosensory: $F=24.1, n=8,7$ for PBS and poly I:C respectively; P6 motor: $F=21.72$, P6 somatosensory: $F=$ 19.59, $n=8,8$ for PBS and poly I:C respectively; P9 motor: $F=$ 12.22, and P9 somatosensory: $F=13.77, n=8,10$ for PBS and poly

neuroblasts is equally distributed across the whole length of the cortex. By P9, while the reduction in density persisted in the somatosensory cortex, much lower difference could be observed in the motor cortex (Fig. 3c, Supplementary Fig. 5e) indicating a differential effect of maternal inflammation on neuroblasts destined for distinct cortical regions.

Cortical GABAergic interneurons are overproduced during embryogenesis, and $\sim 30-40 \%$ of them subsequently undergo apoptosis within the second postnatal week [16].

I:C respectively). d-h Significant reduction in the density of interneurons in the somatosensory but not in the motor cortex at P15. EGFP + cells were quantified in the total cortical thickness as well as in each cortical layer. Panels in $\mathbf{d}$ show representative images of regions used for quantification. Graphs show the total $(\mathbf{e}, \mathbf{f})(n=8$ and 7 for PBS and poly I:C, respectively) as well as the layer-wise $(\mathbf{g}, \mathbf{h})$ reduction in EGFP + cell density. Comparison of means by two-way ANOVA in $\mathbf{a}$-c, Student's $t$-test in $\mathbf{e}$ and $\mathbf{f}$, and $t$-test with Holm-Sidak correction for multiple comparison in $\mathbf{g}$ and $\mathbf{h}$ ) (mean \pm SEM are shown, $* * * p<$ $0.0005)$. Scale bars: $50 \mu \mathrm{m}(\mathbf{d})$

We therefore investigated whether an increase in programmed cell death of cortical interneurons due to maternal inflammation could explain in part the reduction of their number in the cortex of maternal inflammation-affected mice. To this end, we counted activated caspase- 3 and EGFP-expressing cells in several sections covering the motor and somatosensory cortices. At E17.5, P3, and P6, we observed a similar number of activated caspase-3+ $($ Casp3+) as well as EGFP+ Casp3 + cells in control pups 
a

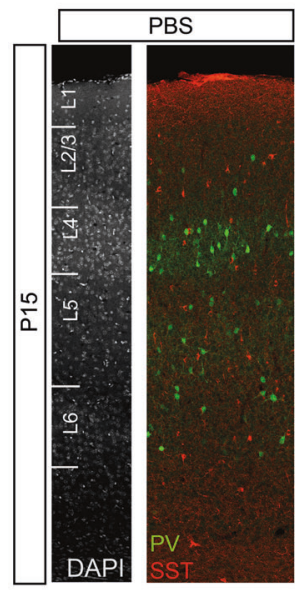

b
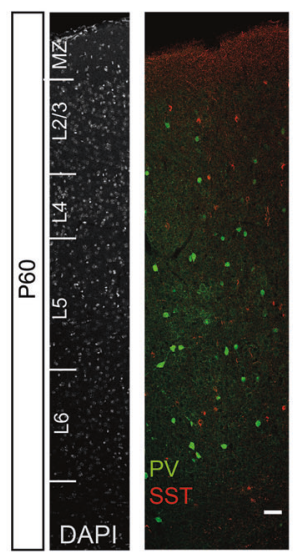

C PV cells $/ \mathrm{mm}^{2}$
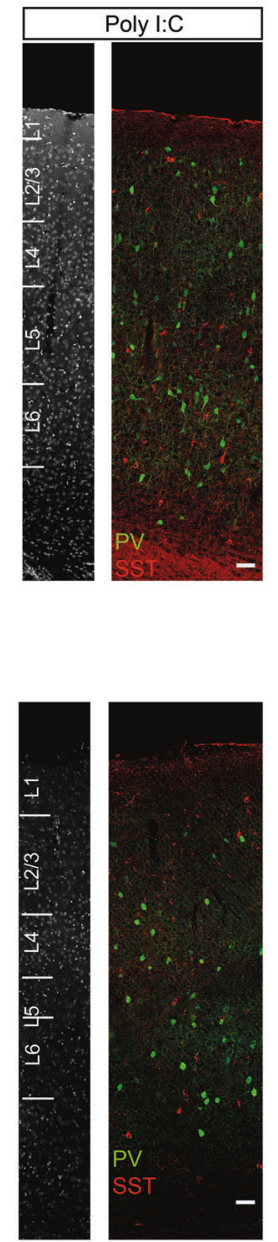

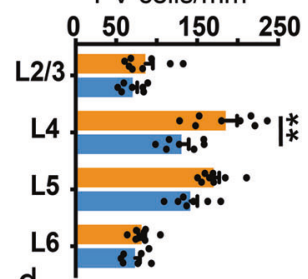

d SST cells $/ \mathrm{mm}^{2}$

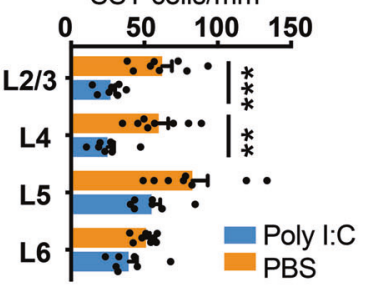

e $\quad \mathrm{PV}$ cells $/ \mathrm{mm}^{2}$

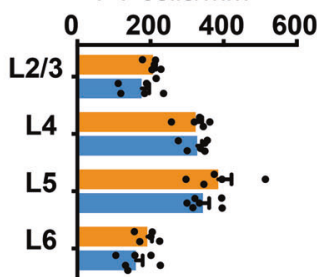

f SST cells $/ \mathrm{mm}^{2}$

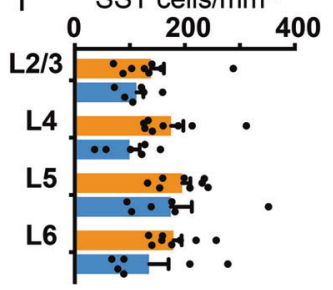

g

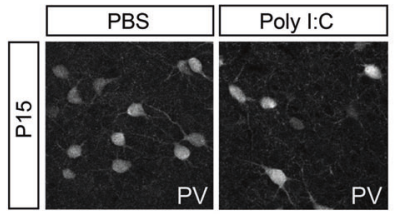

h

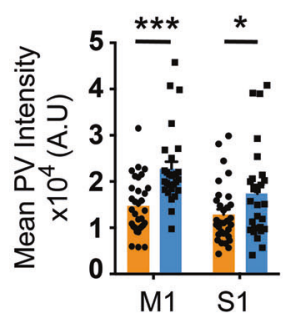

i
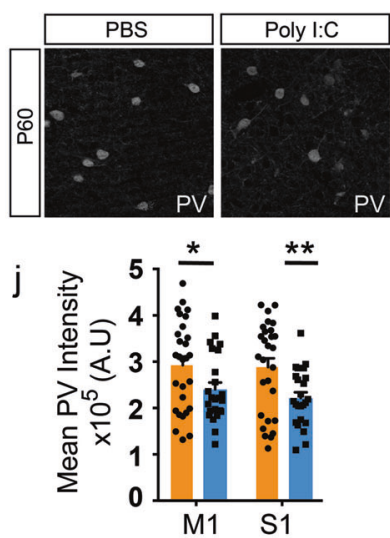

Fig. 4 Interneuron subtype-specific effects of maternal inflammation. a-f Maternal inflammation resulted in a decreased density of PV and SST expressing interneurons at P15 but not at P60. Panels show representative images from $\mathrm{P} 15$ (a) and $\mathrm{P} 60$ (b). A significant decrease in $\mathrm{PV}+$ interneurons could be seen in $\mathrm{L} 4$, while the difference was more pronounced in L2-4 for SST + interneurons $(\mathbf{c}, \mathbf{d})(n=8$ and 7 for PBS and poly I:C, respectively). An appreciable but not statistically significant decrease could be seen at P60 (e, f). g-j Reciprocal changes in PV expression between P15 and P60 due to maternal inflammation. At P15, PV + interneurons in both motor, and somatosensory cortical

and pups exposed to maternal inflammation (Supplementary Fig. 6a, b). Thus, activation of the maternal immune system during early gestation did not augment apoptosis in the developing cortices of pups exposed to maternal inflammation.

To follow up the effect of maternal inflammation during postnatal brain maturation, we further studied the density and positioning of interneurons at P15. Similar to P9, the density of GAD + interneurons in S1 was severely reduced (Fig. 3d, e), whereas no significant changes were observed for M1 (Fig. 3f). Analysis of the layer-wise distribution of interneurons showed an equal effect of maternal inflammation across the whole cortical length in the regions in maternal inflammation-exposed animals showed an increased expression of PV (h) $(n=8$ and 7 for PBS and poly I:C, respectively) measured by the signal intensity in immunolabeled images (g). By P60 (i), this effect had reversed and instead a decreased expression was observed in both motor and somatosensory cortical regions $(\mathbf{j})(n=6$ each). Statistical analysis by $t$-test with Holm-Sidak correction for multiple comparison in $\mathbf{c}-\mathbf{f}, \mathbf{h}$, and $\mathbf{j}$ (mean \pm SEM are shown, $* p<0.05, * * p<0.005, * * * p<0.0005)$. Scale bars: $50 \mu \mathrm{m}$ (a, b)

somatosensory cortex (Fig. 3g), but lack of reduction in GABAergic interneuron density in the motor cortex (Fig. 3h). We hence show that an acute induction of inflammation in pregnant dams impairs migration and final positioning of GAD + neuroblasts with the effect being more pronounced in the somatosensory than in the motor cortex.

\section{Differential effect of maternal inflammation on distinct subtypes of cortical GABAergic interneurons}

While functional impairment of cortical GABAergic interneurons has been proposed to be the one of the major 


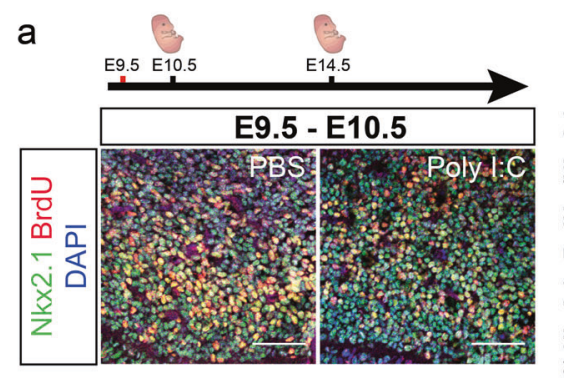

b

E9.5 - E10.5

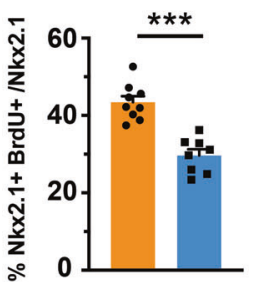

C

E9.5 - E14.5

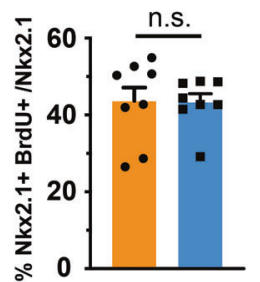

d

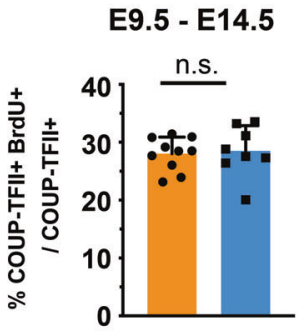

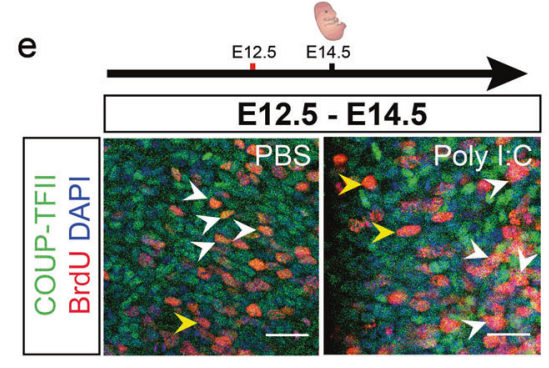

f $\quad E 12.5-E 14.5 \quad g$

E12.5 - E14.5
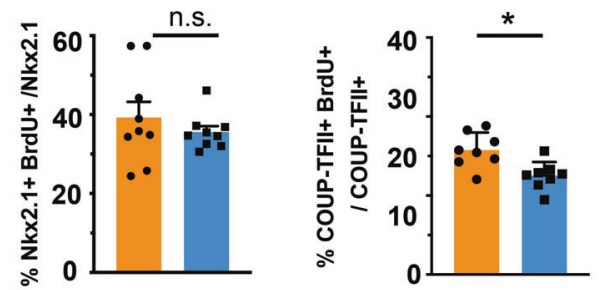

- PBS

Poly I:C

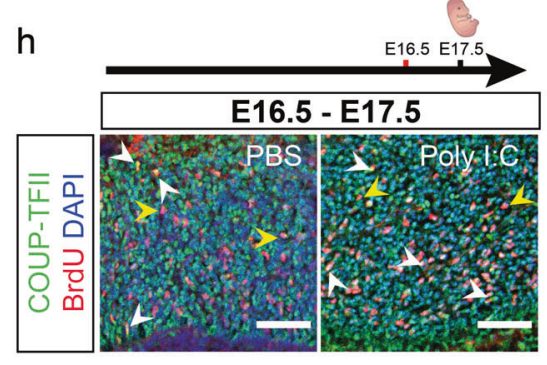

$E 16.5-E 17.5$

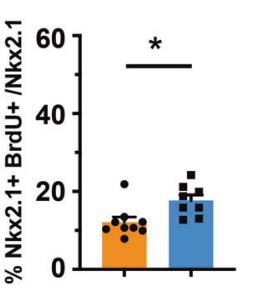

$E 16.5-E 17.5$

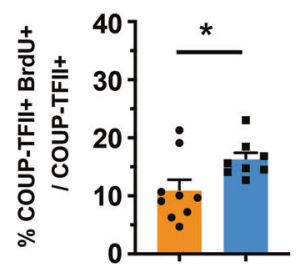

\section{PBS}

Poly I:C

k

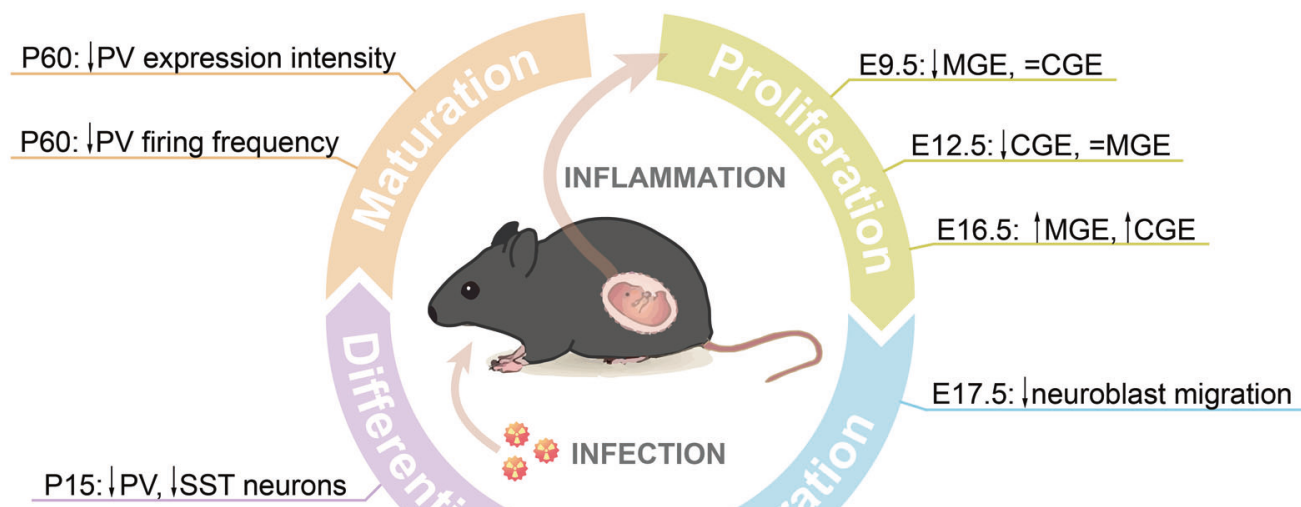

P3-P9: defects in IN distribution

P15: |GAD-EGFP neurons

factors contributing to imbalance between excitation and inhibition in patients with schizophrenia and other psychiatric disorders, most of the attention has been directed towards dysfunction of $\mathrm{PV}+\mathrm{GABAergic}$ interneurons $[24,59,60]$. However, cortical GABAergic interneurons are highly heterogeneous, and PV+ interneurons represent only one class of interneurons [8]. Therefore, the differential impact of maternal immune activation on development and maturation of various interneuron subtypes remains to be elucidated.

To this end, we studied the effect of maternal inflammation on the organization of the four largest interneuron populations, i.e., those that express PV, SST and VIP and $\mathrm{CR}$. In the somatosensory cortex, the previously observed decrease in the density of GAD + interneurons stemmed from a decrease in both $\mathrm{PV}+$ and SST + interneurons 
Fig. 5 Acute and developmental stage-dependent impairment of progenitor proliferation due to maternal inflammation. a-c Maternal inflammation had acute effect on proliferation of MGE-derived Nkx2.1+ progenitors. Dams were injected with poly I:C at E9.5 and embryos assessed at E10.5 after a 2-h BrdU pulse-chase (a, b). Quantification of proliferating Nkx2.1+ BrdU+ cells showed that maternal inflammation resulted in reduction in $\mathrm{Nkx} 2.1+$ proliferating cells at E10.5 (b) ( $n=9$ and 8 for PBS and poly I:C, respectively) that did not sustain until E14.5 (c). COUP-TFII+ progenitors remained unaffected when studied at E14.5 (d). e-g Dams were injected with poly I:C at E12.5 and embryos assessed at E14.5 after a 2-h BrdU pulse-chase. Proliferation of CGE-derived COUP-TFII + progenitors was reduced after maternal inflammation at $\mathrm{E} 12.5(\mathbf{e}, \mathbf{g})(n=9$ and 8 for PBS and poly I:C, respectively) that correlated with the later neurogenic window of CGE-derived progenitors. In contrast, proliferation of $\mathrm{Nkx} 2.1+$ MGE-derived progenitors was unaffected at E14.5 (f). h-j Late maternal inflammation at E16.5 led to an increase in proliferation of both COUP-TFII+ $(\mathbf{h}, \mathbf{j})$ and Nkx2.1+ (i) progenitors $(n=8-9$ for each PBS and Poly I:C). k Schematic representation of the findings in this manuscript. Maternal inflammation affects interneuron development at multiple stages such as proliferation, migration, differentiation and maturation leading to increased vulnerability to mental disorders. Central artwork was modified from [89]. Comparison of means by Student's $t$-test in $\mathbf{b}-\mathbf{d}, \mathbf{f}, \mathbf{g}, \mathbf{I}$, and $\mathbf{j}$ ) (mean \pm SEM are shown, $* p<0.05, * * p<0.005, * * * p<0.0005$ ). Scale bars: $50 \mu \mathrm{m}(\mathbf{a}, \mathbf{e , ~ h})$

(Fig. 4a-f). Interestingly, there was a clear layer-specific effect of maternal inflammation in the distribution of $\mathrm{PV}+$ and SST + interneurons. Thus, the decrease in number of $\mathrm{PV}+$ interneurons was restricted to layer 4 at P15 (Fig. 4c), whereas the reduction of SST+ interneurons was more pronounced across all layers, albeit statistically significant only in layers 2-4 at P15 (Fig. 4d). At P60, the densities of $\mathrm{PV}+$ and $\mathrm{SST}+$ interneurons were not statistically significant between maternal inflammation-affected and control animals even though an appreciable difference could be seen in the case of L4 SST + interneurons (Fig. 4e, f). This is likely caused by the nonuniform expansion of cortical areas during synaptogenesis and gliogenesis [61]. In the motor cortex, there was no significant effect on distribution of interneuron subtypes at P15 and P60 (data not shown). Similar numbers of PV or SST+ interneurons were also observed in the prefrontal cortical areas [62], namely cingulate, prelimbic, and secondary motor cortex (M2), of PBS and poly I:C-exposed mice (Supplementary Fig. 7a-c). There was no effect of maternal inflammation on the distribution of CGE-derived VIP + and $\mathrm{CR}+/ \mathrm{SST}-$ interneurons at P15 (Supplementary Fig. 7d-f).

In addition to the reduced interneuron density, a reduction in PV expression in the cortex and hippocampus has been described in several studies for adult mice [21]. While we confirmed that maternal poly I:C exposure led to a similar reduction in PV expression in both $\mathrm{M} 1$ and $\mathrm{S} 1$ cortical regions at $\mathrm{P} 60$, surprisingly, $\mathrm{PV}+$ interneurons in both regions had an excess of PV expression at P15 (Fig. $4 \mathrm{~g}-\mathrm{j}$, see Supplementary Fig. 8 for PV expression levels in the whole cortex at P60). As PV is an important calcium binding protein in neurons [63], and its expression positively correlates with neuronal activity in the developing cortex [64], the developmental impairment in calcium buffering capacity in PV-expressing neurons might contribute to their functional impairment. Previous studies have shown maternal immune activation to be detrimental to $\mathrm{PV}+$ interneurons of the medial prefrontal cortex leading to decreased GABAergic transmission onto pyramidal neurons [22]. We thus assessed the morphology and functional properties of $\mathrm{PV}+$ interneurons in the superficial layers of the somatosensory cortex in control and animals prenatally exposed to maternal inflammation at P53-57. PV+ interneurons were labeled by Cre-recombinase dependent tdTomato expression in Ai9 reporter mouse $\left(\mathrm{PV}^{\mathrm{Cre}} ; \mathrm{Ai} 9\right)$ and were assessed for intrinsic electrophysiological properties using whole-cell patch-clamp recordings of fluorescent neurons in acute brain slices. The maximum firing frequency of $\mathrm{PV}+$ interneurons was reduced in poly I:Cexposed animals (Supplementary Fig. 9a, b) reflecting an overall slower spiking rate due to maternal immune inflammation. At smaller current injections, no difference could be seen (Supplementary Fig. 9c). In addition, the resting membrane potential and input resistance in poly I:Cexposed animals was not found to be significantly different in comparison with controls (Supplementary Fig. 9d, e). To investigate if the changes in electrophysiological properties are accompanied by anatomical changes, we analyzed the morphology of biocytin-filled PV+ interneurons (Supplementary Fig. 9f). There was no significant effect of maternal inflammation on the length of dendritic filaments (Supplementary Fig. 9g) and branch depth (Supplementary Fig. 9h). However, Sholl analysis of the reconstructed interneurons showed a significant reduction in overall dendritic tree complexity of the maternal inflammation-affected PV+ interneurons (Supplementary Fig. 9i). These results indicate that acute maternal immune response affects morphology and electrophysiology of GABAergic interneurons.

\section{Timing of maternal inflammation affects discreet pools of interneuron progenitors}

One of the most remarkable effects of maternal inflammation on development of the GABAergic interneurons was the decrease in the density of EGFP + neuroblasts as early as E14.5 when maternal immune activation was induced at E9.5 (Fig. 2b, c). As cortical GABAergic interneurons are generated by precursor cells mainly in the MGE and CGE between E9.5 and E18.5 [12, 13], acute maternal inflammation might affect the generation of GABAergic interneurons. To investigate this, GAD67-EGFP pregnant mice were injected with poly I:C at E9.5, E12.5, and E16.5 and proliferating cells were labeled using a 2 -h pulse of the 
thymidine analog, BrdU, at E10.5, E14.5, and E17.5, respectively (Fig. 5a-j). By co-labeling interneuron precursors for BrdU along with Nkx2.1 (MGE-specific marker $[34,65]$ ) or COUP-TFII (CGE-specific marker [66]), we sought to ascertain differences in proliferation of interneuron precursors due to maternal inflammation.

By injecting poly I:C at E9.5 and labelling progenitor proliferation at E10.5, we found an $\sim 25 \%$ decrease in the percentage of $\mathrm{Nkx} 2.1+$ precursors that were co-labeled with BrdU in embryos exposed to maternal inflammation in comparison with unexposed mice (Fig. 5a, b), which correlates very well with a decrease in the number of MGEderived PV+ and SST + interneurons in the postnatal cortex. In contrast, both exposed and unexposed mice showed equal rates of proliferating $\mathrm{Nkx} 2.1+$ progenitors five days post treatment, i.e., at E14.5 (Fig. 5c). This indicates an acute effect of maternal inflammation on the cycling of interneuron progenitors. Furthermore, in keeping with the acute response of progenitors, maternal inflammation exhibited no effect on the proliferation of COUP-TFII+ progenitors over the same period (Fig. 5d), which might be explained by the fact that the majority of CGE-derived interneurons are born after E12.5 [12]. This correlated with the lack of effect on CGE-derived VIP + and $\mathrm{CR}+/ \mathrm{SST}-$ interneurons in the somatosensory cortex at P15 (Supplementary Fig. 7d-f), thus indicating a subtype-specific effect of maternal inflammation on interneuron progenitors.

To investigate whether a later induction of maternal inflammation would affect both CGE-derived (born mainly between E12.5 and E18.5 [12]) and MGE-derived interneurons, we injected dams with poly I:C at E12.5. As with the previous analysis, a 2-h pulse of $\mathrm{BrdU}$ was given before collecting the embryonic brains for analysis at E14.5 (Fig. 5e). Interestingly, we found no difference in the cycling Nkx2.1+ progenitor population at this stage between the two groups (Fig. 5f). On the other hand, we observed an $\sim 20 \%$ decrease in COUP-TFII+ progenitors that were co-labeled with BrdU (Fig. 5g). Despite the decrease in proliferation during embryogenesis, there were no significant reduction in the number of CGE-derived $\mathrm{VIP}+$ and $\mathrm{CR}+/ \mathrm{SST}-$ interneurons in the somatosensory cortex at P15, although we noticed that L5 VIP+ interneurons are virtually absent in maternal inflammationaffected offspring (Supplementary Fig. 10a-c). Such minor effects can be explained by the relatively low production of VIP + and CR + subtypes at E12.5 [13]. In line with our hypothesis of a developmental stage-dependent effect of poly I:C, we did not observe a change in the number of PV + and SST + interneurons due to poly I:C exposure at E12.5 (Supplementary Fig. 10d, e). We also demonstrated that the effect of maternal inflammation was confined to the SVZ, since quantification of $\mathrm{BrdU}+$ proliferating cells in the ventricular zones of MGE and CGE after poly I:C injections at E9.5 and E12.5, respectively, did not show any significant differences from PBS injections (Supplementary Fig. 11a, b).

Finally, as CGE interneuron production peaks at E16.5 [12], we injected poly I:C at this stage in order to capture the maximal effect of maternal inflammation on CGEderived interneurons (Fig. 5h). Surprisingly, and in contrast with the earlier time points, $24 \mathrm{~h}$ later (i.e., at E17.5), we found a marked increase in dividing Nkx2.1+ MGE and COUP-TFII + progenitors $(\sim 37 \%$ and $\sim 70 \%$, respectively) (Fig. 5i, j) indicating that maternal inflammation has an opposing effect at later stages of embryonic development. Therefore, our results show that progenitors of GABAergic interneurons exhibit differential vulnerability to maternal inflammation that depends on the stage of embryonic brain development.

\section{Discussion}

Despite significant evidence showing that maternal immune activation during pregnancy leads to cognitive dysfunction in the offspring that might be triggered by excitation-inhibition imbalance, little is known about how the development of inhibitory system in the brain is affected. Here we show that maternal immune activation results in multiple "hits" on development of GABAergic neurons, thus affecting proliferation of precursors, migration and positioning of neuroblasts as well as their maturation (see summary in Fig. 5k).

Genetic and environmental factors that affect cortical development provide important insights into the cellular and molecular basis of neurodevelopmental disorders. Available epidemiological and clinical findings [67] point towards a link between maternal infections during gestation and increased risk of developing a mental disorder due to impaired cortical development. While the precise mechanism of action is elusive, an increase in maternal cytokine levels is thought to lead to the transmission of maternal inflammation to the fetal brain [3]. However, the developmental changes occurring upon maternal inflammation in the fetal brain and during postnatal brain maturation have been understudied owing to ethical and technical challenges in humans. Thus, rodent models of maternal inflammation provide an excellent alternative to study the effects of an acute inflammatory insult on brain development. Accordingly, poly I:C and lipopolysaccharide (LPS) have emerged as two popular molecules that mimic viral and bacterial infections respectively and robustly activate the maternal immune system. In this study, we mimicked maternal viral infection by injecting poly $\mathrm{I}: \mathrm{C}$ at various developmental time points and followed the development of cortical GABAergic neurons in the offspring brains. Strikingly, in 
spite of an acute immune response, we revealed that the effect was both immediate, i.e., affecting proliferation of precursors of interneurons, and long-lasting, i.e., affecting migration of neuroblasts and maturation of cortical GABAergic neurons during late embryonic or early postnatal brain development.

One of our major findings is that the effect of maternal inflammation has a differential effect on GABAergic interneuron subtypes, highlighting subtype-specific vulnerability of neurons to maternally-derived stimuli. Hitherto, studies have revealed a convergent effect of genetic and environmental schizophrenia-related insults on $\mathrm{PV}+$ interneurons [21, 24]. However, while PV+ interneurons are crucial in synchronizing spike timing via gamma-oscillations, suppression of their activity alone has been found insufficient to reproduce schizophrenia-like phenotype in genetic or environmental mouse models of schizophrenia [68]. We show here a differential impact of maternal inflammation on interneuron subtypes and cortical regions that goes beyond $\mathrm{PV}+$ interneurons. GABAergic interneurons in the somatosensory cortex were more affected in comparison with their counterparts in the motor cortex. Furthermore, the impact on PV+ interneurons was localized specifically to layer 4 and could be traced as early as P15, suggesting a specific developmental impairment. Importantly, we found a significant impact of maternal inflammation on SST + interneurons, which were affected not only in layer 4 as $\mathrm{PV}+$ interneurons but also in layer $2 / 3$ despite both subtypes being derived from MGE-derived Nkx2.1+ progenitors. The overlap in layer 4 is of significance as SST + interneurons in this layer target mainly PV + fastspiking interneurons unlike in layer $2 / 3$ where pyramidal neurons are the primary targets [69]. Selective ablation of $\mathrm{SST}+$ interneurons during development has been shown to arrest the maturation of $\mathrm{PV}+$ interneurons in layer 5/6 suggesting an early role for SST + interneurons in PV+ interneuron maturation [70]. Moreover, the decrease in MGE-derived interneuron populations might disrupt transient translaminar networks [71, 72] that modulate subsequent functional maturation of cortical circuits.

The effect of maternal inflammation at E9.5 on MGEderived $\mathrm{PV}+$ and $\mathrm{SST}+$ interneurons in the offspring can be observed as early as E10.5, due to decreased proliferation of MGE-derived Nkx2.1+ progenitors. However, this effect was acute with proliferation returning to wild-type levels by E14.5. This immediate and short-lived effect of maternal inflammation is in line with previous reports on cortical progenitors in an LPS model $[27,73]$. Contrary to the effect on MGE-derived subtypes, we found no change in CGE-derived VIP + and $\mathrm{CR}+/ \mathrm{SST}-$ interneurons in animals exposed to maternal inflammation at E9.5, and neither CGE-derived COUP-TFII+ progenitors nor VIP + and $\mathrm{CR}+/ \mathrm{SST}$ - interneurons in the mature cortex were affected. This is ostensibly due to a later "birthdate" of CGE-derived interneurons between E12.5 and E18.5 [12]. However, even though the effect on CGE-derived COUPTFII + progenitors could be discerned by inducing inflammation at E12.5, we did not observe a significant decrease in VIP+ interneurons in somatosensory cortex at P15. This can be explained by the low relative production of VIP+ interneurons at E12.5 ( 5\%) [12] or that other but not somatosensory cortical areas are affected. In contrast, inducing maternal inflammation at E16.5 resulted in a marked increase in cycling MGE and CGE progenitors. Such variability of outcomes of maternal inflammation highlights selective vulnerability of interneuron progenitor pools that likely depends on expression of distinct transmembrane and intracellular signaling components at each stage of development.

It can hence be appreciated that the time of insult coupled with cellular or genetic vulnerabilities can produce varying outcomes on interneuron subtypes. Timing of the developmental insult will can determine which developmental processes in the cortex are affected, leading to variation in the cognitive phenotypes and severity of the affected offspring. Indeed, it has been shown that a difference in the timing of maternal inflammation leads to distinct behavioral dysfunctions [39, 40] (see also [74] for review). Over the course of cortex development, maternal inflammation could interfere with: (1) interneuron precursor proliferation, (2) neuronal migration and positioning between cortical areas and within cortical layers, and (3) neuronal maturation and circuit connectivity. Our data show that acute maternal inflammation affects multiple stages of interneuron development. In addition to the impact on precursor proliferation, described above, we show that migration of interneurons into the dorsal cortex was impaired as early as E14.5. The effect of maternal inflammation on the distribution of cortical GABAergic interneurons was maintained at E17.5 but could only be seen in the superficial cortical bins. This could be due to a greater proportion of GAD+ neuroblasts in maternal inflammation-exposed embryos migrating laterally and further work using time-lapse microscopy will be important to clarify the mechanism of this impairment. Likewise, while analysis of early postnatal stages showed a decreased density of GAD+ neuroblasts in regions of the developing cortex that correspond to both motor and somatosensory cortices, by P15 the effect persisted only in the somatosensory cortex. This suggests a differential regional impairment of cortical interneurons. Despite this, perinatal reduction in the number of GAD + neuroblasts in the motor cortex might still affect the maturation of early cortical circuits and have functional outcomes that could not be measured in this study.

The effect of maternal inflammation on different stages of interneuron development (proliferation, migration and 
maturation) might be independent of one another or effects on neuronal migration and maturation might stem from the proliferation deficit. Although the major effect of maternal inflammation has been attributed to an acute increase in levels of pro-inflammatory molecules, the contribution of some low scale but significant chronic effects cannot be excluded. Furthermore, late embryonic inflammation can potentially affect the migration of neuroblasts directly and also those neuroblasts that start to mature in the cortical plate. Most probably, the effect of maternal inflammation on neuronal migration and maturation is complex and consists of direct and indirect components. Likewise, less pronounced differences in the number/distribution of $\mathrm{PV}+$ and $\mathrm{SST}+$ interneurons in adult offspring in comparison with early postnatal stage might be explained by a maturational delay of MGE-derived interneurons that stems from disturbed proliferation and direct chronic post-inflammatory effects.

Functionally, PV + interneurons in our model showed a decreased maximum firing frequency in the absence of changes in input resistance. The maximum firing frequency in PV+ interneurons has been described to increase during postnatal development $[75,76]$, thus suggesting a maturational delay of $\mathrm{PV}+$ interneurons in our study due to maternal inflammation. This maturational delay is also corroborated by decrease in dendritic complexity of maternal inflammation-affected PV+ interneurons. Alterations in cellular properties of GABAergic interneurons can alter the perisomatic inhibition of pyramidal neurons thus altering the excitation-inhibition balance in the neocortex [76]. Furthermore, human studies show widespread changes in neonatal cortical connectivity due to increase in the level of maternal pro-inflammatory cytokines [77, 78]. Importantly, GABAergic interneurons are necessary for maturation of early cortical circuits that provide the foundation for adult cortical connectivity [71, 72]. Thus, maturational delay of GABAergic interneurons due to maternal inflammation can have broad effect on cognitive properties of the affected offspring. In particular, this might lead to reduced cortical plasticity and in turn impaired learning and memory in the older mice.

While this study focuses on GABAergic interneurons, maternal inflammation can also have an effect on populations of pyramidal neurons in the cortex [27, 79]. Furthermore, thalamic and subplate neurons are also likely to be affected at early gestational stages since they are born between E10 and E12 [80, 81]. A disruption of either of these neuronal populations could impact the maturation of GABAergic interneurons and hamper their functional integration into cortical circuits $[82,83]$, thus adding to the overall effect of maternal inflammation on development of GABAergic interneurons.
In order to mitigate litter-to-litter variations due to maternal inflammation, recent guidelines have advocated for considering each litter as an experimental unit [84]. However, in this study we observed a greater within-litter than inter-litter variability. We thus sampled more than one offspring per litter (and studied at least 3 litters per condition). In addition, we used an LMM analysis to appropriately represent this variation while considering the litter as a source of variation. This provided a better depiction of the range of effect of maternal inflammation on offspring.

The poly I:C model of maternal inflammation presents high construct validity and parallels the effects seen in humans [3, 74]. Based on translation of brain development milestones, the early time point of induction (E9.5) overlaps with the first trimester of human pregnancy [47] as well as with the onset of neurogenesis of cortical GABAergic interneurons [85]. Given the significant evidence for impairment of cortical GABAergic interneurons in human postmortem brain studies of patients with mental illness [86-88], the dysfunction of developmental programs for GABAergic interneurons might have causative effect on cognitive phenotype of the patients. Further studies of GABAergic circuitry development in environmental and genetic mouse models mimicking human developmental insults will reveal commonalities and differences in the affected subtypes of GABAergic interneurons across all developmental processes. Such data will provide mechanistic insight into the etiology of human neurodevelopmental disorders and will identify therapeutic windows during brain development when impairments in GABAergic circuitry can be corrected.

Acknowledgements We are grateful to Prof. Yuchio Yanagawa (Gunma University, Japan) for sharing the GAD67-EGFP knock-in mouse line. We also wish to thank Dr Yasuko Antoku for assistance with microscopy, Johanna Meichsner (University of Mainz) for help with statistics, and members of the Khodosevich lab for constructive discussions relating to this study. The work was supported by the Novo Nordisk Foundation Hallas-Møller Investigator grant (NNF16OC0019920), Lundbeck-NIH Brain Initiative grant (20172241), and DFF-Forskningsprojekt1 (8020-00083B) to KK and German Research Foundation (DFG) grant within the Collaborative Research Center (SFB) 1080 "Molecular and Cellular Mechanisms of Neural Homoeostasis" to JvE. NAV is supported by BRIDGETranslational Excellence Programme fellowship funded by Novo Nordisk Foundation (NNF18SA0034956).

Author contributions This study was conceived by NAV, MP-N, UP, and KK. NAV and MP-N performed the poly I:C injections and downstream experiments including design, collection, analysis, and interpretation of data. JG, DW, SM, and DG-G collected and analyzed specific developmental stages. UP performed and analyzed IL-6 ELISA. IK performed and analyzed the western blotting for PV. MKM and JvE performed and analyzed electrophysiology. KSH contributed to the design, execution and analysis of behavioral experiments. NAV and KK jointly wrote the paper. All authors read and approved the manuscript. 


\section{Compliance with ethical standards}

Conflict of interest The authors declare that they have no conflict of interest.

Publisher's note Springer Nature remains neutral with regard to jurisdictional claims in published maps and institutional affiliations.

Open Access This article is licensed under a Creative Commons Attribution 4.0 International License, which permits use, sharing, adaptation, distribution and reproduction in any medium or format, as long as you give appropriate credit to the original author(s) and the source, provide a link to the Creative Commons license, and indicate if changes were made. The images or other third party material in this article are included in the article's Creative Commons license, unless indicated otherwise in a credit line to the material. If material is not included in the article's Creative Commons license and your intended use is not permitted by statutory regulation or exceeds the permitted use, you will need to obtain permission directly from the copyright holder. To view a copy of this license, visit http://creativecommons. org/licenses/by/4.0/.

\section{References}

1. Bale TL, Baram TZ, Brown AS, Goldstein JM, Insel TR, McCarthy MM, et al. Early life programming and neurodevelopmental disorders. Biol Psychiatry. 2010;68:314-9.

2. Schmitt A, Malchow B, Hasan A, Falkai P. The impact of environmental factors in severe psychiatric disorders. Front Neurosci. 2014;8:19.

3. Meyer U. Developmental neuroinflammation and schizophrenia. Prog Neuropsychopharmacol Biol Psychiatry. 2013;42:20-34.

4. Bronson SL, Bale TL. The placenta as a mediator of stress effects on neurodevelopmental reprogramming. Neuropsychopharmacology. 2016;41:207-18.

5. Giovanoli S, Engler H, Engler A, Richetto J, Voget M, Willi R, et al. Stress in puberty unmasks latent neuropathological consequences of prenatal immune activation in mice. Science. 2013;339:1095-9.

6. Choi GB, Yim YS, Wong H, Kim S, Kim H, Kim SV, et al. The maternal interleukin-17a pathway in mice promotes autism-like phenotypes in offspring. Science. 2016;351:933-9.

7. Kim S, Kim H, Yim YS, Ha S, Atarashi K, Tan TG, et al. Maternal gut bacteria promote neurodevelopmental abnormalities in mouse offspring. Nature. 2017;549:528-32.

8. Rudy B, Fishell G, Lee S, Hjerling-Leffler J. Three groups of interneurons account for nearly $100 \%$ of neocortical GABAergic neurons. Dev Neurobiol. 2011;71:45-61.

9. Batista-Brito R, Fishell G. The developmental integration of cortical interneurons into a functional network. Curr Top Dev Biol. 2009;87:81-118.

10. Gelman DM, Marin O. Generation of interneuron diversity in the mouse cerebral cortex. Eur J Neurosci. 2010;31:2136-41.

11. Nord AS, Pattabiraman K, Visel A, Rubenstein JL. Genomic perspectives of transcriptional regulation in forebrain development. Neuron. 2015;85:27-47.

12. Miyoshi G, Hjerling-Leffler J, Karayannis T, Sousa VH, Butt SJ, Battiste $\mathrm{J}$, et al. Genetic fate mapping reveals that the caudal ganglionic eminence produces a large and diverse population of superficial cortical interneurons. J Neurosci. 2010;30:1582-94.

13. Miyoshi G, Butt SJ, Takebayashi H, Fishell G. Physiologically distinct temporal cohorts of cortical interneurons arise from telencephalic Olig2-expressing precursors. J Neurosci. 2007; 27:7786-98.
14. Ang ES Jr., Haydar TF, Gluncic V, Rakic P. Four-dimensional migratory coordinates of GABAergic interneurons in the developing mouse cortex. J Neurosci. 2003;23:5805-15.

15. Tanaka DH, Yanagida M, Zhu Y, Mikami S, Nagasawa T, Miyazaki J, et al. Random walk behavior of migrating cortical interneurons in the marginal zone: time-lapse analysis in flatmount cortex. J Neurosci. 2009;29:1300-11.

16. Southwell DG, Paredes MF, Galvao RP, Jones DL, Froemke RC, Sebe JY, et al. Intrinsically determined cell death of developing cortical interneurons. Nature. 2012;491:109-13.

17. Pfisterer U, Khodosevich K. Neuronal survival in the brain: neuron type-specific mechanisms. Cell Death Dis. 2017;8:e2643.

18. Wray NR, Ripke S, Mattheisen M, Trzaskowski M, Byrne EM, Abdellaoui A, et al. Genome-wide association analyses identify 44 risk variants and refine the genetic architecture of major depression. Nat Genet. 2018;50:668-81.

19. Hunt RF, Girskis KM, Rubenstein JL, Alvarez-Buylla A, Baraban SC. GABA progenitors grafted into the adult epileptic brain control seizures and abnormal behavior. Nat Neurosci. 2013;16: $692-7$.

20. Zuckerman L, Rehavi M, Nachman R, Weiner I. Immune activation during pregnancy in rats leads to a postpubertal emergence of disrupted latent inhibition, dopaminergic hyperfunction, and altered limbic morphology in the offspring: a novel neurodevelopmental model of schizophrenia. Neuropsychopharmacology. 2003;28:1778-89.

21. Jiang Z, Cowell RM, Nakazawa K. Convergence of genetic and environmental factors on parvalbumin-positive interneurons in schizophrenia. Front Behav Neurosci. 2013;7:116.

22. Canetta S, Bolkan S, Padilla-Coreano N, Song LJ, Sahn R, Harrison NL, et al. Maternal immune activation leads to selective functional deficits in offspring parvalbumin interneurons. Mol Psychiatry. 2016;21:956-68.

23. Richetto J, Calabrese F, Riva MA, Meyer U. Prenatal immune activation induces maturation-dependent alterations in the prefrontal GABAergic transcriptome. Schizophr Bull. 2013;40: 351-61.

24. Steullet P, Cabungcal JH, Coyle J, Didriksen M, Gill K, Grace $\mathrm{AA}$, et al. Oxidative stress-driven parvalbumin interneuron impairment as a common mechanism in models of schizophrenia. Mol Psychiatry. 2017;22:936-43.

25. Semple BD, Blomgren K, Gimlin K, Ferriero DM, NobleHaeusslein LJ. Brain development in rodents and humans: Identifying benchmarks of maturation and vulnerability to injury across species. Prog Neurobiol. 2013;106-7:1-16.

26. Li WY, Chang YC, Lee LJ, Lee LJ. Prenatal infection affects the neuronal architecture and cognitive function in adult mice. Dev Neurosci. 2014;36:359-70.

27. Stolp HB, Turnquist C, Dziegielewska KM, Saunders NR, Anthony DC, Molnar Z. Reduced ventricular proliferation in the foetal cortex following maternal inflammation in the mouse. Brain. 2011;134:3236-48.

28. Richetto J, Calabrese F, Riva MA, Meyer U. Prenatal immune activation induces maturation-dependent alterations in the prefrontal GABAergic transcriptome. Schizophr Bull. 2014;40: 351-61.

29. Amin H, Marinaro F, De Pietri Tonelli D, Berdondini L. Developmental excitatory-to-inhibitory GABA-polarity switch is disrupted in 22q11.2 deletion syndrome: a potential target for clinical therapeutics. Sci Rep. 2017;7:15752.

30. Sauer J-F, Strüber M, Bartos M. Impaired fast-spiking interneuron function in a genetic mouse model of depression. Elife. 2015;4:566.

31. Lee E, Lee J, Kim E. Excitation/inhibition imbalance in animal models of autism spectrum disorders. Biol Psychiatry. 2017;81: 838-47. 
32. Ascoli GA, Alonso-Nanclares L, Anderson SA, Barrionuevo G, Benavides-Piccione R, Burkhalter A, et al. Petilla terminology: nomenclature of features of GABAergic interneurons of the cerebral cortex. Nat Rev Neurosci. 2008;9:557-68.

33. Pearce BD. Schizophrenia and viral infection during neurodevelopment: a focus on mechanisms. Mol Psychiatry. 2001;6:634-46.

34. Butt SJ, Sousa VH, Fuccillo MV, Hjerling-Leffler J, Miyoshi G, Kimura S, et al. The requirement of Nkx2-1 in the temporal specification of cortical interneuron subtypes. Neuron. 2008;59: 722-32.

35. Alcántara S, Ruiz M, Arcangelo GD, Ezan F, Lecea LDe, Curran $\mathrm{T}$, et al. Regional and cellular patterns of reelin mRNA expression in the forebrain of the developing and adult mouse. J Neurosci. 1998;18:7779-99.

36. Miller BJ, Culpepper N, Rapaport MH, Buckley P. Prenatal inflammation and neurodevelopment in schizophrenia: a review of human studies. Prog Neuropsychopharmacol Biol Psychiatry. 2013;42:92-100.

37. Giovanoli S, Notter T, Richetto J, Labouesse MA, Vuillermot S, Riva MA, et al. Late prenatal immune activation causes hippocampal deficits in the absence of persistent inflammation across aging. J Neuroinflammation. 2015;12:221.

38. Smolders S, Smolders SM, Swinnen N, Gartner A, Rigo JM, Legendre $\mathrm{P}$, et al. Maternal immune activation evoked by polyinosinic:polycytidylic acid does not evoke microglial cell activation in the embryo. Front Cell Neurosci. 2015;9:301.

39. Meyer U, Nyffeler M, Engler A, Urwyler A, Schedlowski M, Knuesel I, et al. The time of prenatal immune challenge determines the specificity of inflammation-mediated brain and behavioral pathology. J Neurosci. 2006;26:4752-62.

40. Meyer U, Nyffeler M, Yee BK, Knuesel I, Feldon J. Adult brain and behavioral pathological markers of prenatal immune challenge during early/middle and late fetal development in mice. Brain Behav Immun. 2008;22:469-86.

41. Tamamaki N, Yanagawa Y, Tomioka R, Miyazaki J, Obata K, Kaneko T. Green fluorescent protein expression and colocalization with calretinin, parvalbumin, and somatostatin in the GAD67-GFP knock-in mouse. J Comp Neurol. 2003;467:60-79.

42. Khodosevich K, Lazarini F, von Engelhardt J, Kaneko H, Lledo $\mathrm{PM}$, Monyer H. Connective tissue growth factor regulates interneuron survival and information processing in the olfactory bulb. Neuron. 2013;79:1136-51.

43. Le Magueresse C, Alfonso J, Khodosevich K, Arroyo Martin AA, Bark C, Monyer H. 'Small axonless neurons': postnatally generated neocortical interneurons with delayed functional maturation. J Neurosci. 2011;31:16731-47.

44. Hougaard KS, Jackson P, Kyjovska ZO, Birkedal RK, De Temmerman P-J, Brunelli A, et al. Effects of lung exposure to carbon nanotubes on female fertility and pregnancy. A study in mice. Reprod Toxicol. 2013;41:86-97.

45. Garcia-Gonzalez D, Khodosevich K, Watanabe Y, Rollenhagen A, Lubke JHR, Monyer H. Serotonergic projections govern postnatal neuroblast migration. Neuron. 2017;94:534-49.

46. Zorrilla EP. Problems (and possibilities) to developmentalists. Dev Psychobiol. 1997;30:141-50.

47. Workman AD, Charvet CJ, Clancy B, Darlington RB, Finlay BL. Modeling transformations of neurodevelopmental sequences across mammalian species. J Neurosci. 2013;33:7368-83.

48. Meyer U. Prenatal poly(I_C) exposure and other developmental immune activation models in rodent systems. Biol Psychiatry. 2014;75:307-15.

49. Smith SE, Li J, Garbett K, Mirnics K, Patterson PH. Maternal immune activation alters fetal brain development through interleukin-6. J Neurosci. 2007;27:10695-702.

50. Richetto J, Massart R, Weber-Stadlbauer U, Szyf M, Riva MA, Meyer U. Genome-wide DNA methylation changes in a mouse model of infection-mediated neurodevelopmental disorders. Biol Psychiatry. 2017;81:265-76.

51. Meyer U, Feldon J, Schedlowski M, Yee BK. Towards an immuno-precipitated neurodevelopmental animal model of schizophrenia. Neurosci Biobehav Rev. 2005;29:913-47.

52. Giovanoli S, Engler H, Engler A, Richetto J, Feldon J, Riva MA, et al. Preventive effects of minocycline in a neurodevelopmental two-hit model with relevance to schizophrenia. Transl Psychiatry. 2016;6:e772.

53. Weber-Stadlbauer U, Richetto J, Labouesse MA, Bohacek J, Mansuy IM, Meyer U. Transgenerational transmission and modification of pathological traits induced by prenatal immune activation. Mol Psychiatry. 2017;22:102-12.

54. Reite M, Teale P, Rojas DC, Benkers TL, Carlson J. Anomalous somatosensory cortical localization in schizophrenia. Am J Psychiatry. 2003;160:2148-53.

55. Teale P, Pasko B, Collins D, Rojas D, Reite M. Somatosensory timing deficits in schizophrenia. Psychiatry Res. 2013;212:73-8.

56. Pascual-Leone A, Manoach DS, Birnbaum R, Goff DC. Motor cortical excitability in schizophrenia. Biol Psychiatry. 2002;52: 24-31.

57. Anderson SA, Eisenstat DD, Shi L, Rubenstein JLR. Interneuron migration from basal forebrain to neocortex: Dependence on Dlx genes. Science. 1997;278:474-6.

58. Tamamaki N, Fujimori KE, Takauji R. Origin and route of tangentially migrating neurons in the developing neocortical intermediate zone. J Neurosci. 1997;17:8313-23.

59. Hashemi E, Ariza J, Rogers H, Noctor SC, Martinez-Cerdeno V. The number of parvalbumin-expressing interneurons is decreased in the medial prefrontal cortex in Autism. Cereb Cortex. 2017;27:1931-43.

60. Georgiev D, Yoshihara T, Kawabata R, Matsubara T, Tsubomoto M, Minabe Y, et al. Cortical gene expression after a conditional knockout of $67 \mathrm{kDa}$ glutamic acid decarboxylase in parvalbumin neurons. Schizophr Bull. 2016;42:992-1002.

61. Hammelrath L, Škokić S, Khmelinskii A, Hess A, van der Knaap $\mathrm{N}$, Staring M, et al. Morphological maturation of the mouse brain: an in vivo MRI and histology investigation. Neuroimage. 2016; 125:144-52.

62. Carlén M. What constitutes the prefrontal cortex? Science. 2017;358:478-82.

63. Celio MR, Heizmann CW. Calcium-binding protein parvalbumin as a neuronal marker. Nature. 1981;293:300-2.

64. Patz S, Grabert J, Gorba T, Wirth MJ, Wahle P. Parvalbumin expression in visual cortical interneurons depends on neuronal activity and TrkB ligands during an Early period of postnatal development. Cereb Cortex. 2004;14:342-51.

65. Du T, Xu Q, Ocbina PJ, Anderson SA. NKX2.1 specifies cortical interneuron fate by activating Lhx6. Development. 2008;135: 1559-67.

66. Kanatani S, Yozu M, Tabata H, Nakajima K. COUP-TFII is preferentially expressed in the caudal ganglionic eminence and is involved in the caudal migratory stream. J Neurosci. 2008;28: 13582-91.

67. Brown AS, Derkits EJ. Prenatal infection and schizophrenia: a review of epidemiologic and translational studies. Am J Psychiatry. 2010;167:261-80.

68. Hamm JP, Peterka DS, Gogos JA, Yuste R. Altered cortical ensembles in mouse models of schizophrenia. Neuron. 2017;94: 153-67.

69. Xu H, Jeong HY, Tremblay R, Rudy B. Neocortical somatostatinexpressing GABAergic interneurons disinhibit the thalamorecipient layer 4. Neuron. 2013;77:155-67.

70. Tuncdemir SN, Wamsley B, Stam FJ, Osakada F, Goulding M, Callaway EM, et al. Early somatostatin interneuron connectivity mediates the maturation of deep layer cortical circuits. Neuron. 2016;89:521-35. 
71. Anastasiades PG, Marques-Smith A, Lyngholm D, Lickiss T, Raffiq S, Katzel D, et al. GABAergic interneurons form transient layer-specific circuits in early postnatal neocortex. Nat Commun. 2016;7:10584.

72. Marques-Smith A, Lyngholm D, Kaufmann AK, Stacey JA, Hoerder-Suabedissen A, Becker EB, et al. A transient translaminar GABAergic interneuron circuit connects thalamocortical recipient layers in neonatal somatosensory cortex. Neuron. 2016;89: 536-49.

73. Oskvig DB, Elkahloun AG, Johnson KR, Phillips TM, Herkenham M. Maternal immune activation by LPS selectively alters specific gene expression profiles of interneuron migration and oxidative stress in the fetus without triggering a fetal immune response. Brain Behav Immun. 2012;26:623-34.

74. Reisinger S, Khan D, Kong E, Berger A, Pollak A, Pollak DD. The poly(I:C)-induced maternal immune activation model in preclinical neuropsychiatric drug discovery. Pharm Ther. 2015;149:213-26.

75. Yang J-M, Zhang J, Yu Y-Q, Duan S, Li X-M. Postnatal development of 2 microcircuits involving fast-spiking interneurons in the mouse prefrontal cortex. Cereb Cortex. 2014;24:98-109.

76. Corradini I, Focchi E, Rasile M, Morini R, Desiato G, Tomasoni $\mathrm{R}$, et al. Maternal immune activation delays acid switch in offspring. Biol Psychiatry. 2018;83:680-91.

77. Spann MN, Monk C, Scheinost D, Peterson BS. Maternal immune activation during the third trimester is associated with neonatal functional connectivity of the salience network and fetal to toddler behavior. J Neurosci. 2018;38:2272-17.

78. Rudolph MD, Graham AM, Feczko E, Miranda-Dominguez O, Rasmussen JM, Nardos R, et al. Maternal IL-6 during pregnancy can be estimated from newborn brain connectivity and predicts future working memory in offspring. Nat Neurosci. 2018; 21:765-72.
79. Shin Yim Y, Park A, Berrios J, Lafourcade M, Pascual LM, Soares N, et al. Reversing behavioural abnormalities in mice exposed to maternal inflammation. Nature. 2017;549:482-7.

80. Angevine JB. Time of neuron origin in the diencephalon of the mouse. An autoradiographic study. J Comp Neurol. 1970;139: 129-87.

81. Hoerder-Suabedissen A, Molnár Z. Molecular diversity of earlyborn subplate neurons. Cereb Cortex. 2013;23:1473-83.

82. Kanold PO, Shatz CJ. Subplate neurons regulate maturation of cortical inhibition and outcome of ocular dominance plasticity. Neuron. 2006;51:627-38.

83. Deng R, Kao JPY, Kanold PO. Distinct translaminar glutamatergic circuits to GABAergic interneurons in the neonatal auditory cortex. Cell Rep. 2017;19:1141-50.

84. Kentner AC, Bilbo SD, Brown AS, Hsiao EY, McAllister AK, Meyer U, et al. Maternal immune activation: reporting guidelines to improve the rigor, reproducibility, and transparency of the model. Neuropsychopharmacology 2019;44:245-58.

85. Zecevic N, Hu F, Jakovcevski I. Interneurons in the developing human neocortex. Dev Neurobiol. 2011;71:18-33.

86. Fung SJ, Webster MJ, Sivagnanasundaram S, Duncan C, Elashoff $\mathrm{M}$, Weickert CS. Expression of interneuron markers in the dorsolateral prefrontal cortex of the developing human and in schizophrenia. Am J Psychiatry. 2010;167:1479-88.

87. Rocco BR, DeDionisio AM, Lewis DA, Fish KN. Alterations in a unique class of cortical chandelier cell axon cartridges in schizophrenia. Biol Psychiatry. 2017;82:40-8.

88. Volk DW, Edelson JR, Lewis DA. Altered expression of developmental regulators of parvalbumin and somatostatin neurons in the prefrontal cortex in schizophrenia. Schizophr Res. 2016; 177:3-9.

89. Powell CM. Neuroscience: mum's bacteria linked to baby's behaviour. Nature 2017;549:466-7. 